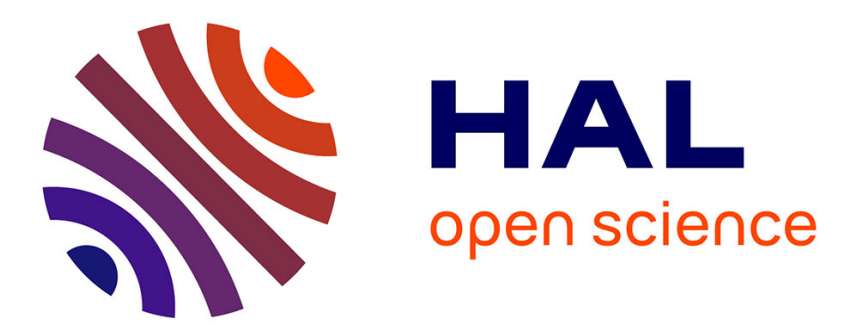

\title{
Non-equilibrium relaxation of hot states in organic semiconductors: Impact of mode-selective excitation on charge transfer
}

\author{
Antonios M Alvertis, Florian A. Y. N. Schröder, Alex W Chin
}

\section{To cite this version:}

Antonios M Alvertis, Florian A. Y. N. Schröder, Alex W Chin. Non-equilibrium relaxation of hot states in organic semiconductors: Impact of mode-selective excitation on charge transfer. Journal of Chemical Physics, 2019, 151 (8), pp.084104. 10.1063/1.5115239 . hal-02377572

\section{HAL Id: hal-02377572 \\ https://hal.sorbonne-universite.fr/hal-02377572}

Submitted on 23 Nov 2019

HAL is a multi-disciplinary open access archive for the deposit and dissemination of scientific research documents, whether they are published or not. The documents may come from teaching and research institutions in France or abroad, or from public or private research centers.
L'archive ouverte pluridisciplinaire HAL, est destinée au dépôt et à la diffusion de documents scientifiques de niveau recherche, publiés ou non, émanant des établissements d'enseignement et de recherche français ou étrangers, des laboratoires publics ou privés. 


\section{Non-equilibrium relaxation of hot states in organic semiconductors: Impact of mode- selective excitation on charge transfer}

Cite as: J. Chem. Phys. 151, 084104 (2019); https://doi.org/10.1063/1.5115239

Submitted: 15 June 2019 . Accepted: 04 August 2019 . Published Online: 23 August 2019

Antonios M. Alvertis (D), Florian A. Y. N. Schröder (D), and Alex W. Chin
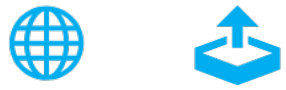

\section{ARTICLES YOU MAY BE INTERESTED IN}

\section{Multiscale approach to electron transport dynamics}

The Journal of Chemical Physics 151, 084105 (2019); https://doi.org/10.1063/1.5112372

Optical spectra in the condensed phase: Capturing anharmonic and vibronic features using dynamic and static approaches

The Journal of Chemical Physics 151, 074111 (2019); https://doi.org/10.1063/1.5114818

Compressing physics with an autoencoder: Creating an atomic species representation to improve machine learning models in the chemical sciences

The Journal of Chemical Physics 151, 084103 (2019); https://doi.org/10.1063/1.5108803

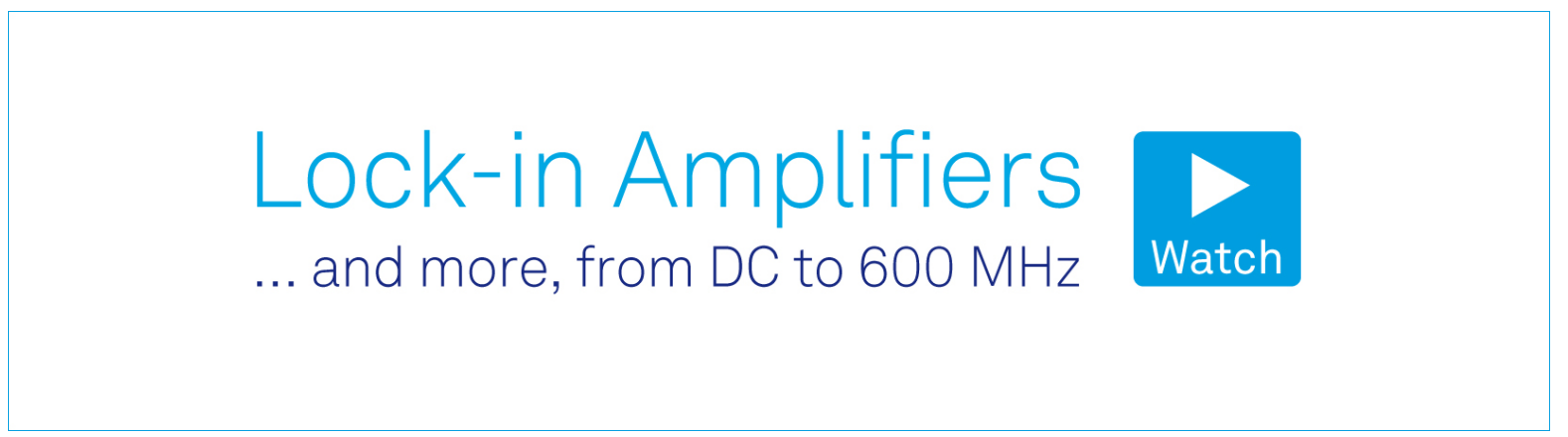

... and more, from DC to $600 \mathrm{MHz}$ Watch 


\title{
Non-equilibrium relaxation of hot states in organic semiconductors: Impact of mode-selective excitation on charge transfer
}

\author{
Cite as: J. Chem. Phys. 151, 084104 (2019); doi: 10.1063/1.5115239 \\ Submitted: 15 June 2019 - Accepted: 4 August 2019• \\ Published Online: 23 August 2019
}

Antonios M. Alvertis, ${ }^{1, a)}$ (D) Florian A. Y. N. Schröder, ${ }^{\text {(D) }}$ and Alex W. Chin ${ }^{2, b)}$

\begin{abstract}
AFFILIATIONS
${ }^{1}$ Cavendish Laboratory, University of Cambridge, J. J. Thomson Avenue, Cambridge CB3 OHE, United Kingdom

${ }^{2}$ CNRS, Institut des NanoSciences de Paris, Sorbonne Université, 4 place Jussieu boite courrier 840,

75252 Paris Cedex 05, France
\end{abstract}

a) Electronic mail: ama80@cam.ac.uk

b) Electronic mail: alex.chin@insp.jussieu.fr

\begin{abstract}
The theoretical study of open quantum systems strongly coupled to a vibrational environment remains computationally challenging due to the strongly non-Markovian characteristics of the dynamics. We study this problem in the case of a molecular dimer of the organic semiconductor tetracene, the exciton states of which are strongly coupled to a few hundreds of molecular vibrations. To do so, we employ a previously developed tensor network approach, based on the formalism of matrix product states. By analyzing the entanglement structure of the system wavefunction, we can expand it in a tree tensor network state, which allows us to perform a fully quantum mechanical time evolution of the exciton-vibrational system, including the effect of 156 molecular vibrations. We simulate the dynamics of hot states, i.e., states resulting from excess energy photoexcitation, by constructing various initial bath states, and show that the exciton system indeed has a memory of those initial configurations. In particular, the specific pathway of vibrational relaxation is shown to strongly affect the quantum coherence between exciton states in time scales relevant for the ultrafast dynamics of application-relevant processes such as charge transfer. The preferential excitation of low-frequency modes leads to a limited number of relaxation pathways, thus "protecting" quantum coherence and leading to a significant increase in the charge transfer yield in the dimer
\end{abstract} structure.

Published under license by AIP Publishing. https://doi.org/10.1063/1.5115239

\section{INTRODUCTION}

Light-harvesting in organic molecules is a fundamental process which has attracted a lot of attention in recent years, its applications ranging from photosynthetic systems ${ }^{1,2}$ to organic solar cells. Following photoexcitation, ultrafast (i.e., femtosecond to picosecond time scale) nonequilibrium dynamics drive the exciton energy transfer in these systems, ${ }^{4}$ underlining the need to move away from the traditional thermodynamic way of thinking, in order to better understand and engineer such processes. In the past decade, the experimental study of organic semiconductors has provided a good understanding of ultrafast processes, through the use of techniques such as pump-push probe spectroscopy ${ }^{5-7}$ and transient absorption microscopy. ${ }^{8,9}$ Several studies have emphasized the role of strong exciton-phonon coupling for driving energy transfer in these systems, ${ }^{10-13}$ indicating the relevance of non-Markovian physics for such phenomena.

Following photoexcitation, one may think of the interaction of an exciton system with the potentially large number of molecular vibrations of organic structures as the interaction of an open quantum system with its (vibrational) environment. The various experimental setups outlined previously allow for preparing different initial quantum states, the time evolution of which can be followed with great precision in the femtosecond time scale. Examples include "hot" states, i.e., exciton states with excess energy pumped into molecular vibrations. ${ }^{14,15}$ The insights generated from such 
works may be generalized to a wide range of open quantum systems, which are relevant for quantum technologies, biology, and areas such as nanoscale quantum heat engines. ${ }^{16-21}$ Furthermore, by formulating the problem within the framework of open quantum system theory, several techniques from these fields of study become available in order to study the nonequilibrium dynamics from a theoretical perspective. Such open quantum system treatments of organic molecules have been used in the past, ${ }^{22,23}$ including techniques ranging from reduced density matrix descriptions (master equations $)^{24-26}$ to complete simulations of system-bath wave functions with methods such as multiconfigurational time-dependent Hartree (MCDTH). ${ }^{27-29}$

However, the theoretical and computational study of these systems poses a significant challenge for several reasons. First, the large number of molecular vibrations often found in organic molecules makes it necessary to account for complicated manybody interactions. The strong exciton-phonon coupling which was mentioned previously further complicates things as traditionally used perturbative approaches are not necessarily applicable in this case. ${ }^{13,30}$ For these reasons, we adopt a tensor network formula$\operatorname{tion}^{31}$ based on the formalism of matrix product states (MPSs), which has previously been used to study the ultrafast dynamics of singlet exciton fission ${ }^{33}$ and exciton-polariton dynamics. ${ }^{34,35}$ This so-called tree tensor network state (TTNS) approach shows strong similarities to the multilayer formulation of $\mathrm{MCTDH}^{36}$ and can capture the full non-Markovian quantum dynamics of a system coupled to a large number of vibrations (few hundreds), without relying on any perturbative approach. Moreover, it efficiently retains all information about the vibrational modes of the environment, enabling us to identify and visualize the often complex chain of environmental processes that drive ultrafast dissipative dynamics.

Here, we use our TTNS formalism to explore the dynamics of systems prepared far from equilibrium such that excess vibrational energy could potentially promote transient excitonic dynamics that lead to new outcomes, which would not occur for band-edge excitation (i.e., no excess vibrational energy). The role of such "hot" states has been a subject of debate in the literature, in particular regarding the process of charge transfer (CT) and separation, in materials used for organic photovoltaics. ${ }^{14,15,37,38}$ However, their importance extends to several temperature-activated processes, such as endothermic singlet exciton fission ${ }^{39}$ and energy transfer in biological systems. ${ }^{40,41}$ Our results indicate that excess energy can have a large impact on the early time scales of nonequilibrium dynamics, leading to very different yields for the process of charge transfer in a molecular dimer of the organic semiconductor tetracene which we focus on in this work. Perhaps most intriguingly, we find that initial quantum states where low-frequency modes are excited preferentially lead to greater charge transfer yields due to robust, mode-generated quantum coherence between the initial exciton and the charge transfer state. Indeed, modes of different frequencies have been discussed to play different roles in the coherent dynamics of charge transfer, ${ }^{42}$ while the electron mobility in a Holstein model has been shown to strongly depend on the initial vibrational preparation. ${ }^{43}$ We relate the behavior observed in our system to the different pathways of vibrational relaxation which the system follows, depending on the energy of the excited vibrations. Overall, we believe that our study suggests a more general principle for engineering the dynamics of open quantum systems and helps open a way to understanding how excess energy, heat generation, and work can be managed in quantum optoelectronic devices. Encouragingly, recent experimental advances in the ultrafast preparation and control of particular vibrational modes in excited states, including the exploitation of ultrastrong light-vibration coupling (polaritons), suggest that the underlying microscopic physics now available in simulations of quantum internal conversion may be tested in the relatively near-term. ${ }^{44,45}$

The structure of this paper is as follows. In Sec. II, we outline the used methods. Section II A provides a general introduction to tensor network methods for studying the dynamics of open quantum systems, as they have been developed both in previous and in this work. Section II B deals with the specifics of applying this formalism to a molecular system, for which it is necessary to obtain its electronic and vibrational properties. The results on the ultrafast dynamics of charge transfer in this molecular system are presented in Sec. III, comparing the cases of having finite or zero excess energy in the system, under different conditions. The conclusions of our work are finally given in Sec. IV.

\section{METHODOLOGY}

\section{A. Tensor networks}

\section{Matrix product states}

In order to develop some intuition for the tree-tensor network state (TTNS) ansatz that we shall use to simulate molecular open system dynamics, we will begin with a simpler illustration using matrix product states (MPSs). A comprehensive review of the properties and uses of MPSs, as well as their fundamental links to density matrix and Wilsonian renormalization group algorithms, can be found in Ref. 32. Let us consider a one dimensional many-body system of $L$ (distinguishable) interacting particles, each of which is localized on a lattice "site" $k$ and has a local (noninteracting) Hilbert space basis $\left\{\left|n_{k}\right\rangle\right\}$ of dimension $d_{k}$. The exact many-body wave function may be formally written in the Kronecker product basis as

$$
|\Psi\rangle=\sum_{\left\{n_{k}\right\}=1}^{d_{k}} \Psi_{n_{1}, \ldots, n_{L}}\left|n_{1}, \ldots, n_{L}\right\rangle,
$$

where $\left|n_{1}, \ldots, n_{L}\right\rangle$ is shorthand for the tensor product $\left|n_{1}\right\rangle$ $\otimes\left|n_{2}\right\rangle \otimes \cdots \otimes\left|n_{L}\right\rangle$, and the sum runs over all possible products of $n_{k}$. The multidimensional array, here referred to as a tensor, $\Psi_{n_{1}, \ldots, n_{L}}$, contains the probability amplitudes for all possible states of the chain. As the number of particles grows, the number of elements stored in this tensor scales as $d_{k}^{L}$ and will quickly become too large for practical computations. This is often called the curse of dimensionality. However, by iteratively applying singular value decomposition (SVD), any $|\Psi\rangle$ may be written as a product of rank three tensors, known as a Matrix Product State (MPS),

$$
\left|\Psi_{M P S}\right\rangle=\sum_{\left\{n_{k}\right\}=1}^{d_{k}} A^{n_{1}} \cdot \ldots \cdot A^{n_{L}}\left|n_{1}, \ldots, n_{L}\right\rangle .
$$

Each of these tensors $A^{n_{k}}$ has dimensions $D_{k-1} \times D_{k}$ x $d_{k}$, with $D_{k-1}$, $D_{k}$ referred to as the left and right bond dimensions and $d_{k}$ referred 
to as the bond dimension of the local Hilbert space. The maximum value of $D_{k}$ encodes the amount of entanglement between neighboring sites. Since for every configuration the tensors must multiply into a scalar, we have the boundary condition $D_{1}=D_{L}=1$. The procedure of writing a many-body wavefunction as an MPS is summarized in Fig. 1. The local Hilbert space of each site may be compressed by using an optimized boson basis $(\mathrm{OBB})^{46,47}$ with $d_{O B B, k} \ll d_{k}$,

$$
A^{n_{k}}=\sum_{\tilde{n}_{k}}^{d_{O B B, k}} A^{\tilde{n}_{k}} V_{n_{k}, \tilde{n}_{k}} .
$$

Once a state has been written as an MPS, any variational optimization or time-evolution may be performed by "sweeping along the chain," i.e., updating one tensor at a time, in a manner similar to density matrix renormalization group (DMRG) approaches. ${ }^{32}$ The MPS approach thus benefits from the reduction of the problem to a sequence of single site problems and the storage and manipulation of a number of matrices that scales linearly with system size. When we account for the overheads due to the contraction of tensors, single value decomposition, etc., the effective scaling becomes cubic in system size, but this is still a vast improvement on the previous exponential scaling. Discussions and bench marking data related to system sizes, convergence, and central processing unit (CPU) times for spin-boson-like systems can be found in Refs. 33 and 47.

\section{Systems interacting with a vibrational environment}

Let us examine the case of a system which is in contact with a vibrational "bath," i.e., a set of oscillators with which it can exchange energy. The Hamiltonian of such a system may be schematically represented as in Fig. 2 (left). Such a system does not have a onedimensional architecture which would allow a one-to-one match between the different components of the Hamiltonian and the MPS tensors. However, even for such a system, the problem may be recast in that of a one-dimensional system using an orthogonal polynomial mapping. ${ }^{48}$ Such an approach has previously been employed to
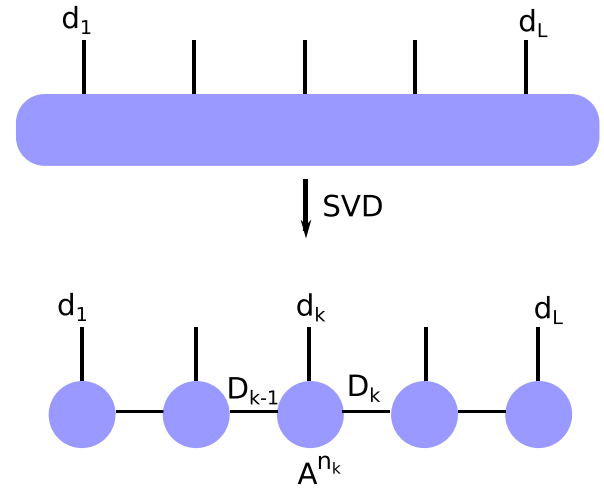

FIG. 1. A Matrix Product State (MPS) is constructed from a many-body wavefunction by applying Singular Value Decomposition (SVD) iteratively.

study the well known Spin Boson Model (SBM) in the case where it is coupled to an environment of oscillators. ${ }^{47,49,50}$ This process is visualized in Fig. 2. The bond dimension between the system tensor and the environment chain $\left(D_{h}\right)$ may in principle be different from the intrachain bond dimension (D).

Similar to the case of the SBM, this approach of representing a system interacting with a bath of oscillators has previously been employed for molecular systems. ${ }^{33}$ However, in such a system, different molecular vibrations can have very different effects on the system states, e.g., only shifting the energies of particular levels or only coupling specific states to each other. It is therefore necessary to separate molecular vibrations into different groups, which in Ref. 33 approximately correspond to different irreducible representations of the molecular point group. However, when there are no obvious molecular symmetries to exploit, this grouping of vibrations may still be performed in a rigorous manner by using the machine learning technique known as k-means++ clustering.

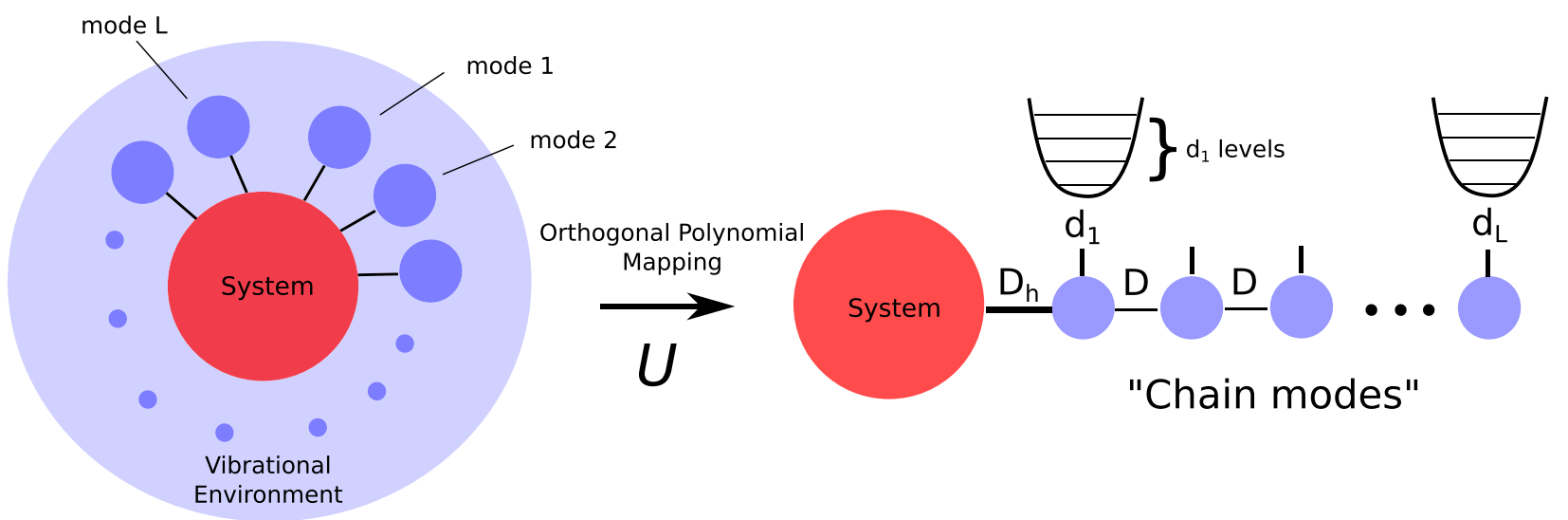

FIG. 2. A system interacting with a set of oscillators (left) may be transformed into a "chain architecture" (right) using an orthogonal polynomial mapping U. The new modes are linear combinations of the initial ones, and we refer to them as "chain modes." 
In order to better understand this procedure, let us work with a linear vibronic Hamiltonian which describes the coupling of the system states to the vibrations,

$$
H^{m, n}=H_{e l}^{m, n}+\sum_{k} W_{k}^{m, n} Q_{k}+\sum_{k} \frac{\hbar \omega_{k}}{2}\left(-\frac{\partial^{2}}{\partial Q_{k}^{2}}+Q_{k}^{2}\right),
$$

where $Q_{k}$ are the dimensionless displacements along normal mode $k$, indicating the contribution of that mode to the molecular deformation. The matrices $W_{k}$ give the first order couplings between the system states due to deformation along mode $k$ and will hence be referred to as vibronic coupling matrices. In terms of creation and annihilation operators,

$$
H=H_{e l}+\sum_{k}\left(W_{k} \frac{a_{k}^{\dagger}+a_{k}}{\sqrt{2}}+\hbar \omega_{k} a_{k}^{\dagger} a_{k}\right) .
$$

By normalizing the coupling matrices $W_{k}$ as

$$
\left(W_{m n}\right)_{k}=\left(\hat{W}_{m n}\right)_{k} \lambda_{k}
$$

the matrix $\left(\hat{W}_{m n}\right)_{k}$ contains the coupling pattern between the system states due to displacement along mode $k$, while the constant $\lambda_{k}$ describes the coupling strength.

For molecular systems, the number of vibrational modes may be of the order of a few hundreds; it is therefore crucial to reduce the computational cost of a time-evolution. By using the machine learning technique $\mathrm{k}-\mathrm{means}++{ }^{51}$ we can identify patterns among the matrices $W_{k}$, splitting them into several groups. To do so, the normalized matrices $\hat{W}_{k}$ are represented as vectors on a highdimensional unit sphere and then projected on two dimensions using $t$-distributed stochastic neighbor embedding ( $t$-SNE) ${ }^{52}$ The k-means++ algorithm assigns a centroid matrix $\bar{W}_{i}$ to each of the created groups $i$, representing the effect of the group of modes on the system. This is visualized in Fig. 3 for the case of the molecular system studied in this work (see below). The individual modes still retain their different coupling strengths $\lambda_{k}$ but now act as one "environment," which can be transformed into a one-dimensional chain of oscillators using an orthogonal polynomial mapping.
Hence, the linear vibronic Hamiltonian may be written in its final form,

$$
H_{s t a r}=H_{e l}+\sum_{i} \bar{W}_{i} \sum_{k} \lambda_{i, k} \frac{b_{i, k}^{\dagger}+b_{i, k}}{\sqrt{2}}+H_{c, i},
$$

where $H_{c, i}$ is the Hamiltonian of the transformed vibrational modes of chain $i$. The creation and annihilation operators $b_{k}^{\dagger}, b_{k}$ now refer to the "chain modes" which result from the orthogonal polynomial transformation of the original system vibrations.

\section{Time evolution and tree tensor network states}

Once we apply an orthogonal polynomial mapping to every vibrational environment of the linear vibronic Hamiltonian and bring it in the form of Eq. (7), it may be schematically represented as in the left hand side of Fig. 4. We represent the many-body wavefunction as a tensor network which resembles this same architecture; this is essentially several MPSs connected to each other through the central system (red) tensor. We refer to this wavefunction as the "star MPS."

The time-evolution of a wavefunction written as a star MPS in Fig. 4 (left) may be done using the time-dependent variational principle. $^{33,53}$ In short, each tensor is sequentially time-evolved using a local effective Hamiltonian, which is constructed by contracting the full many-body Hamiltonian with all MPS tensors which are not currently being updated. Unfortunately, this star MPS suffers a curse of dimensionality: due to being connected to each other through the central tensor, all possible environment configurations are entangled, making its size to scale exponentially with the number of environment chains.

In order to address the exponential scaling problem, the star MPS tensor might be further decomposed into a number of smaller, entanglement renormalization tensors (ER nodes), which describe the interenvironment entanglement. ${ }^{54}$ The entanglement of various bipartitions of the system tensor is quantified by calculating the corresponding von Neumann entropy. The tensors are then connected into a tree structure, the tree tensor network state (TTNS), according to the coupling structure of the star Hamiltonian, in order to minimize the total von Neumann entropy. To find the optimal
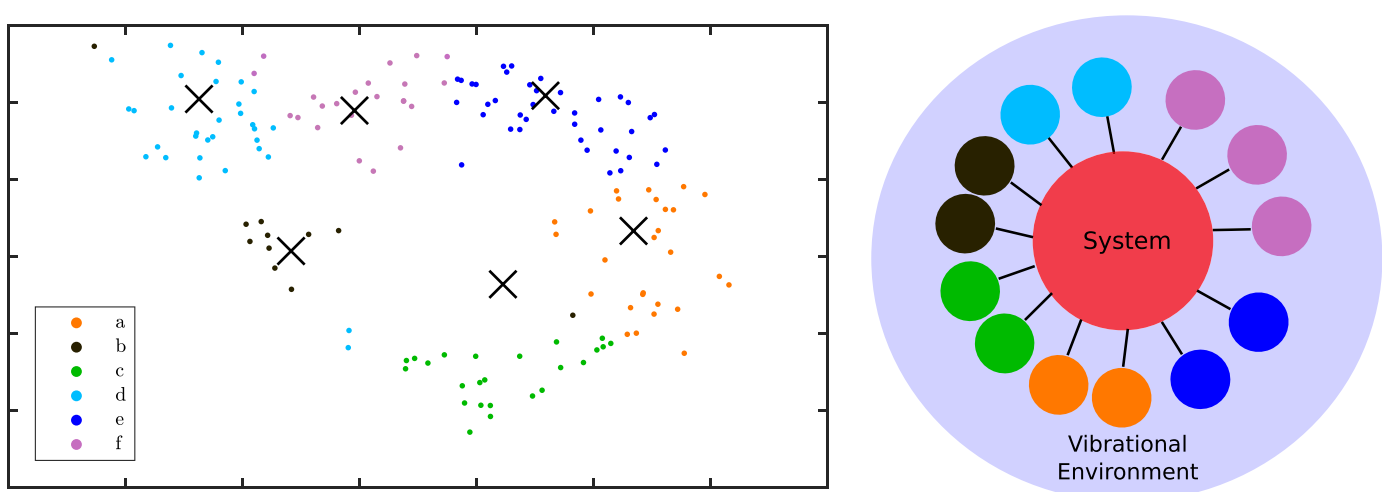

FIG. 3. Clustering of molecular vibrations using k-means++. ${ }^{51}$ We find that a minimum of six clusters are required to obtain converged dynamics for our system of study. The modes belonging to different clusters are represented using different colors. 


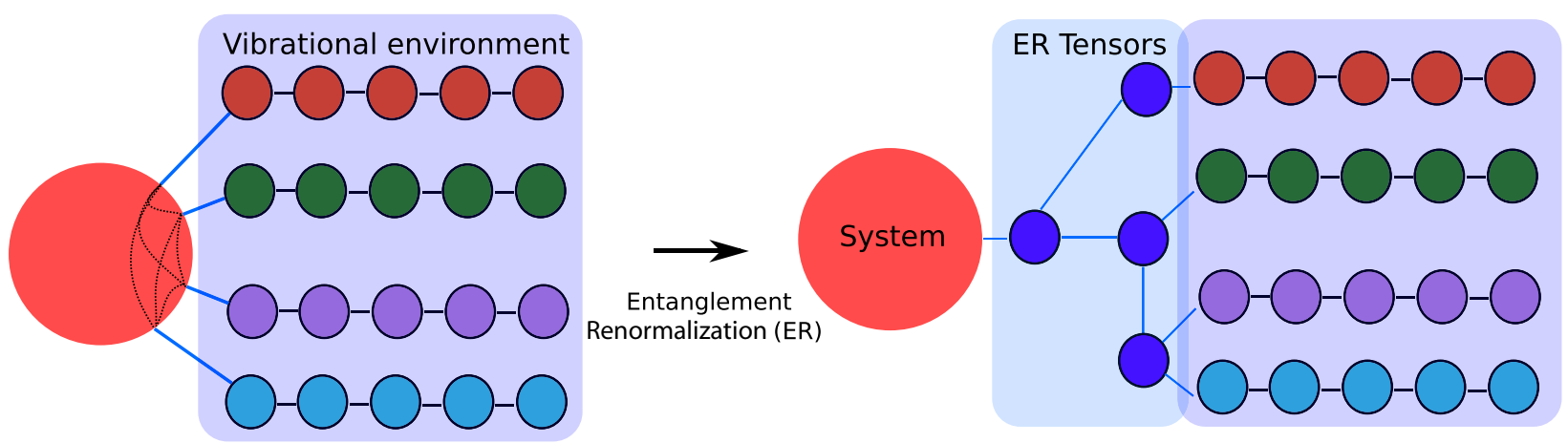

FIG. 4. By means of an orthogonal polynomial transformation, k-means++ clustering, and entanglement renormalization (ER), we transform the initial wavefunction of the system in a vibrational bath into a tree tensor network state (TTNS).

tree structure, an initial simulation with the star MPS is performed, allowing us to assess the entropy of various possible tree structures and construct the one with the minimal value. The TTNS obtained through this analysis allows for using the smallest possible values for bond dimensions, in order to capture the entanglement of the system. The size of this tensor network now scales linearly, which is a significant improvement compared to the initial exponential scaling. The process for determining the TTNS is summarized in Fig. 4.

At this stage, it is important to remember that the timeevolution is performed on the system after the application of an orthogonal polynomial mapping was applied to each of the vibrational environments. Therefore, in order to obtain the timedependent observables of the original system, an inverse mapping is required in each case.

\section{Constructing initial states}

Finally, we would like to discuss a few ways of constructing an initial TTNS, which correspond to various different physical initial conditions. Since in this work we are concerned with vibrational relaxation and the effects of adding excess energy in the system, i.e., initially exciting not only the "system" but also certain vibrations of the "bath," let us consider a few possible ways of defining a vibrationally excited TTNS as our initial state.

a. Band-edge excitation. By band-edge excitation, we mean an initial state where a system state is excited; however, no excess energy is added to the vibrational bath. By denoting each tensor within our MPS representation as $A\left(D_{k-1}, D_{k}, d_{k}\right)$, we set $A(1,1,1)=1$ and all other values to zero. Therefore, from the sum of Eq. (2), only the term with all vibrational modes in their ground state, and the system in the first of the two states, survives. We remind at this point that $d_{k}$ denotes the bond dimension of the local Hilbert space of site $k$, while the bond dimensions $D_{k-1}, D_{k}$ encode the entanglement which the MPS can describe between neighboring sites. For tensors representing vibrations, we find that a maximum value of $d_{k}=40$ leads to converged dynamics for all cases examined in this work. This means that we allow each site to accommodate up to 40 Fock states. For the special case of the two-state exciton system $d=2$. We also find that for the intrachain and system-environment entanglement (see Fig. 2), the bond dimensions $\mathrm{D}=35$ and $\mathrm{D}_{\mathrm{h}}=50$ produce converged results, respectively (refer to the Appendix for convergence tests).

b. Exciting a "chain mode." The most straightforward way of adding excess energy to the system is by adding a quantum of energy to one of the tensors $l$ representing vibrations on the right hand side of Fig. $4: A_{l}(1,1,1)=0$ and $A_{l}(1,1,2)=1$, i.e., we populate the first excited state (second Fock state, $d_{k}=2$ ) of the mode at site $l$. However, one has to bear in mind that these are not the original, physical vibrations of the system, but the ones resulting from the orthogonal polynomial transformation. The energy corresponding to one quantum of such a chain mode may be derived analytically, as done in Ref. 47. Since the excitation of these "chain modes" does not correspond to physical reality, we do not pursue this avenue but rather develop two different methods for adding energy to the original molecular vibrations.

c. Incoherent (quantized) excitation of a normal mode. Let us start by describing the example case of defining an initial MPS, which describes a state with one quantum of excitation added to mode $k$ of the original system. The orthogonal polynomial transformation discussed previously may be used to write the annihilation (or creation) operator of "chain mode" $i$, in terms of the annihilation operators of the physical normal modes,

$$
b_{i}=\sum_{k} U_{i k} a_{k}
$$

Since the mapping $U$ is unitary, it is straightforward to obtain the operators of the original modes in terms of chain modes,

$$
a_{k}=\sum_{i} U_{k i}^{*} b_{i}
$$

Therefore, the state with one quantum on mode $k$ is a linear combination of states with one quantum on the various chain modes $i$ and coefficients given by the inverse of the orthogonal polynomial mapping $\left(U_{k i}^{*}=U_{k i}\right)$,

$$
|1\rangle_{k}=a_{k}^{\dagger}|0\rangle=\sum_{i} U_{k i} b_{i}^{\dagger}|0\rangle .
$$


To write this in the form of an MPS, one has to realize a way in which the sum over all possible configurations of Eq. (2) produces exactly the state of Eq. (10). All the configurations describing states which do not contribute to this sum, for example, states with two quanta on different modes, should vanish. To achieve this for the case of one quantum, one may define the tensors within the chain as

$$
A_{i}(:,:, 1)=\left[\begin{array}{ll}
1 & 0 \\
0 & 1
\end{array}\right], A_{i}(:,:, 2)=\left[\begin{array}{rr}
0 & U_{i k} \\
0 & 0
\end{array}\right] .
$$

Since matrices need to multiply into a scalar for each configuration, the last site $L$ of a chain is always represented by column vectors,

$$
A_{L}(:, 1)=\left[\begin{array}{l}
0 \\
1
\end{array}\right], A_{L}(:, 2)=\left[\begin{array}{l}
U_{i k} \\
0
\end{array}\right] .
$$

The first site of the chain is the tensor representing the system, which is correspondingly represented as a row vector of dimensions $1 \times 2$ in this case. In this manner, if site $i$ is in its excited state represented by the second matrix of Eq. (11), then if any other site is in its excited state the probability of encountering this state is zero since the product of two such matrices is zero. Only states with a single excited chain mode multiplying chain modes in their ground state will survive in the sum of Eq. (2) and will have a prefactor of $U_{i k}$. Therefore, we obtain exactly the state of Eq. (10). By considering the last site of the chain $L$, this becomes rather easy to see, by asking the question of which $2 \times 2$ matrix needs to operate on each column vector in order for the product to vanish/survive.

Before generalizing to $\mathrm{N}$ quanta excitation, let us consider one more example case of adding two quanta on mode $k$,

$$
|2\rangle_{k}=\frac{1}{\sqrt{2}}\left(a_{k}^{\dagger}\right)^{2}|0\rangle=\frac{1}{\sqrt{2}}\left(\sum_{i} U_{k i} b_{i}^{\dagger}\right)^{2}|0\rangle .
$$

Other than the possibility of having two quanta on chain mode $i$, we now also need to allow for two different chain modes $i$ and $j$ accommodating a quantum each, as described by the cross terms which appear once we take the square of the sum of Eq. (13). It is easier to start from the MPS tensors of the last chain site,

$$
A_{L}(:,:, 1)=\left[\begin{array}{l}
0 \\
0 \\
1
\end{array}\right], A_{L}(:,:, 2)=\left[\begin{array}{l}
0 \\
U_{i k} \\
0
\end{array}\right], A_{L}(:,:, 3)=\left[\begin{array}{c}
\frac{U_{i k}^{2}}{\sqrt{2}} \\
0 \\
0
\end{array}\right],
$$

which allow for the possibility of having two, one, or zero quanta on the last chain mode of a chain. The vector $A_{L}(:,:, 3)$ needs to only give nonzero entries once it encounters matrices of other sites in their ground state, $A_{L}(:,:, 2)$ in the case where the other sites have one or zero quanta, and $A_{L}(:,:, 1)$ in all cases. Therefore, for the intermediate sites $i$ of the chain,

$$
\begin{gathered}
A_{i}(:,:, 1)=\left[\begin{array}{lll}
1 & 0 & 0 \\
0 & 1 & 0 \\
0 & 0 & 1
\end{array}\right], A_{i}(:,:, 2)=\left[\begin{array}{ccr}
0 & U_{i k} & 0 \\
0 & 0 & U_{i k} \\
0 & 0 & 0
\end{array}\right], \\
A_{i}(:,:, 3)=\left[\begin{array}{rrr}
0 & 0 & \frac{U_{i k}^{2}}{\sqrt{2}} \\
0 & 0 & 0 \\
0 & 0 & 0
\end{array}\right] .
\end{gathered}
$$

From the way we defined the initial MPS for the cases of one and two quanta, the reader may already start to identify a pattern going toward higher excitations. We generalize for the case of $N$ quanta excitation by defining the nonzero entries of the initial MPS as

$$
A_{L}(N-m+2,1, m)=\frac{U_{i k}^{m-1}}{\sqrt{(m-1) !}},
$$

where $m$ runs over all integers from 1 to $N+1$. For intermediate chain sites $i$,

$$
A_{i}(1, N+1, N+1)=\frac{U_{i k}^{m-1}}{\sqrt{(m-1) !}}, A_{i}(l, l, 1)=1,
$$

where 1 obtains integer values from 1 to $N+1$. Then,

$$
A_{i}(N, N+1, m)=\frac{U_{i k}^{m-1}}{\sqrt{(m-1) !}}, A_{i}(1, m, m)=\frac{U_{i k}^{m-1}}{\sqrt{(m-1) !}},
$$

and finally,

$$
A_{i}(1, m, m)=\frac{U_{i k}^{m-1}}{\sqrt{(m-1) !}}, A_{i}(1+j, m+j, m)=\frac{U_{i k}^{m-1}}{\sqrt{(m-1) !}},
$$

with $j$ obtaining all integer values from 1 to $N-m$. As opposed to the case of exciting a coherent state, which is a linear combination of different Fock states and is described in Subsection II A 4 d, the process outlined here only results in the excitation of one Fock state. To differentiate between the two, we refer to this case as "incoherent" excitation, as opposed to the coherent excitation of Subsection II A 4 d.

d. Coherent (continuous) excitation of a normal mode. Rather than adding a set number of quanta to a selected normal mode, one can displace a vibration continuously by setting its initial dimensionless displacement $Q$ appearing in the linear vibronic Hamiltonian of Eq. (4) to a finite value $Q=\alpha$. However, how to do this when working in the chain representation might not be immediately obvious.

In order to create a displaced state $|\alpha\rangle$ from the vacuum state $|0\rangle$, one may apply the displacement operator,

$$
D(\alpha)=\exp \left(\alpha a^{\dagger}-\alpha^{*} a\right),
$$

as $|\alpha\rangle=D(\alpha)|0\rangle$. The state $|\alpha\rangle$ is commonly referred to as a coherent state. The expectation values of its position and momentum oscillate just like those of a classical harmonic oscillator initially displaced by $\alpha$, and it corresponds to the state excited by a coherent laser pulse.

One may use the inverse orthogonal polynomial mapping of Eq. (9) and write the above displacement operator for mode $k$ in terms of the chain modes,

$$
D_{k}=\prod_{i} \exp \left(\alpha \cdot U_{k i}\left(b_{i}^{\dagger}-b_{i}\right)\right) .
$$

Therefore, we only have to apply this operator to the MPS defined for the case of band-edge excitation above, in order to obtain an initial state where mode $k$ is displaced by $\alpha$. Each of the exponential 
operators appearing in the product of Eq. (21) may be written as a $d_{i} \times d_{i}$ square matrix. By writing the coherent state in the basis of Fock states

$$
|\alpha\rangle=e^{-\frac{|\alpha|^{2}}{2}} \sum_{n} \frac{\alpha^{n}}{\sqrt{n !}}|n\rangle
$$

one realizes that for larger $n$ values, the terms of the sum become increasingly small. It is therefore possible to truncate a number of terms, corresponding to reducing the second dimension of the matrix representation of the exponential operators of Eq. (21). This is similar to the transformation of the Fock basis into the optimized boson basis through the matrices $V$ appearing in Eq. (3), therefore, we denote these truncated exponential matrices as having dimensions $d \times d_{O B B}$. We find that choosing $d_{O B B}=20$ allows for correctly describing initial coherent states with displacements of up to $\alpha=6$ while still maintaining a reasonable computational cost.

\section{B. Studied system}

We study a covalent dimer of the organic semiconducting molecule tetracene, shown in Fig. 5. Tetracene is a prototypical organic semiconductor, best known for its ability to efficiently undergo an ultrafast process known as singlet fission, ${ }^{56,57}$ despite the process being endothermic. ${ }^{58,59}$ For covalently linked systems as the one at hand, it is common practice to introduce mesityl side groups for solubility reasons. ${ }^{60}$ We therefore refer to this ditetracene-mesityl system as DT-Mes in short.

We optimize the geometry of DT-Mes using density functional theory at the B3LYP, cc-pVDZ level. We find that the molecule assumes an orthogonal configuration due to the steric repulsion between the hydrogen atoms on the two tetracene monomers. The molecular vibrations are obtained at the same level of theory. Concerning the excited states of this system, we perform calculations $^{61,62}$ at the ground state geometry based on the theory of Parr, Pariser and Pople (PPP), which have successfully been used. ${ }^{56,63,64}$ Because the Mesityl side groups break the conjugation of the molecular structures, we truncate them in order to be able to apply PPP theory. However, both excited states considered in this study are localized on the tetracene monomers (see below), so this truncation has a negligible effect on the excited state energies.

Due to the approximate $C_{2}$ symmetry of the molecule, we find that the excited states transform either as $B$ or $A$ irreducible representations of the point group. There are two kinds of excitations in such a system, visualized in Fig. 5: a local exciton (LE), i.e., a state where a bound electron-hole pair is localized on a monomer, and a charge transfer (CT) exciton, where the electron and hole are localized on different monomers. Charge transfer excitons are particularly relevant for light-harvesting applications as they are the precursor to a charge separated state. In terms of the excitons visualized in Fig. 5, the symmetry adapted states may be written as

$$
\begin{aligned}
& \left|\mathrm{LE}_{A}\right\rangle=\frac{1}{\sqrt{2}}\left(\left|\mathrm{LE}_{m}\right\rangle+\left|\mathrm{LE}_{n}\right\rangle\right) \quad \text { (A symmetry), } \\
& \left|\mathrm{LE}_{B}\right\rangle=\frac{1}{\sqrt{2}}\left(\left|\mathrm{LE}_{m}\right\rangle-\left|\mathrm{LE}_{n}\right\rangle\right) \quad \text { (B symmetry), } \\
& \left|\mathrm{CT}_{A}\right\rangle=\frac{1}{\sqrt{2}}\left(\left|\mathrm{CT}_{m \rightarrow n}\right\rangle+\left|\mathrm{CT}_{n \rightarrow m}\right\rangle\right) \quad \text { (A symmetry), } \\
& \left|\mathrm{CT}_{B}\right\rangle=\frac{1}{\sqrt{2}}\left(\left|\mathrm{CT}_{m \rightarrow n}\right\rangle-\left|\mathrm{CT}_{m \rightarrow n}\right\rangle\right) \quad \text { (B symmetry), }
\end{aligned}
$$

where the indices $m$ and $n$ refer to the individual tetracene monomers.

The LE state of $B$ symmetry is the only state with a finite oscillator strength among the above. Since we are concerned with the dynamics following photoexcitation of the bright state, we simplify the problem by only including the bright LE state, which from now on we refer to simply as LE. From the calculation of the vibronic couplings (see below) between LE and the two CT states, we find that it is predominantly coupled to the CT state of $B$ symmetry. We therefore choose $\mathrm{CT}_{B}$ as the second excited state of our model. This two-state model is sufficient to obtain insights into the mechanism of vibrational relaxation, which is the aim of this work. However, in general, the $A$ symmetry states can be of importance for other photophysical processes as singlet fission.

The CT state lies $0.17 \mathrm{eV}$ above the LE state; hence, charge transfer from the bright exciton is an endothermic process. At the ground state geometry, there is a small but finite electronic coupling between the LE and CT states, and in the basis $\{|\mathrm{LE}\rangle,|\mathrm{CT}\rangle\}$, the electronic Hamiltonian may be written as

$$
H_{e l}=\left[\begin{array}{lr}
2.39 & -0.014 \\
-0.014 & 2.56
\end{array}\right](\mathrm{eV}) .
$$

The fact that the electronic Hamiltonian is not diagonal means that the adiabatic states of the system are linear combinations of the LE

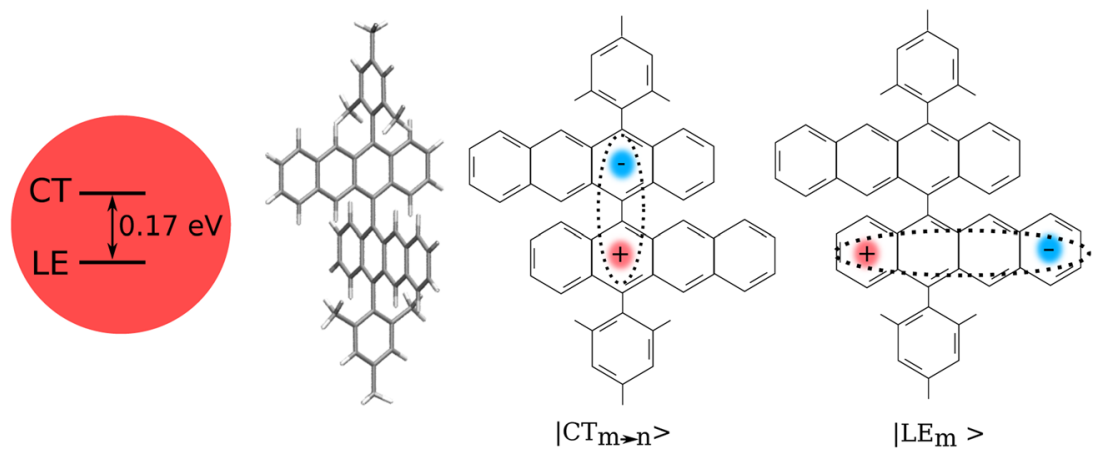

FIG. 5. Studied system includes an electronic system of two excitons, a local exciton (LE) and a charge transfer exciton (CT), coupled to an environment of 156 oscillators. The oscillators correspond to molecular normal modes. The indices $m$ and $n$ refer to the individual tetracene monomers. 
and CT states. However, since this coupling is small, the first and second excited states are of almost pure LE and CT characteristics, respectively. In this basis, the reduced density matrix of the system may be written as

$$
\rho_{n m}=\left[\begin{array}{ll}
\rho_{\mathrm{LE}, \mathrm{LE}} & \rho_{\mathrm{LE}, \mathrm{CT}} \\
\rho_{\mathrm{LE}, \mathrm{CT}} & \rho_{\mathrm{CT}, \mathrm{CT}}
\end{array}\right],
$$

where the diagonal entries give the probability of finding the system in the LE or CT state, while the off-diagonal element is the quantum coherence between the two excitons.

The two excitons are coupled to a bath of oscillators, which are the molecular normal modes. Out of the 210 vibrational modes of the truncated DT-Mes structure, we include a total of 156 in our model. These are modes with frequencies between $100 \mathrm{~cm}^{-1}$ and $1600 \mathrm{~cm}^{-1}$, i.e., we exclude very high-frequency modes, mostly $\mathrm{C}-\mathrm{H}$ stretches, which are not relevant for the dynamics, as well as very slow low-frequency modes, which are not strongly displaced in the ultrafast time scale. In addition, low-frequency modes are known to be strongly anharmonic ${ }^{65}$ and cannot be treated within the harmonic approximation, on which the linear vibronic model relies.

In order to obtain the coupling between the electronic states upon displacement of the vibrational modes, i.e., the vibronic couplings, we displace the molecular structure in the positive and negative directions of each normal mode $\mathbf{q}$ and recalculate the electronic states using PPP calculations. The coupling element between two states is then given by

$$
W_{m n}=\frac{\left\langle m\left|\mathscr{H}\left(\mathbf{r}_{o}+h \mathbf{q}\right)\right| n\right\rangle-\left\langle m\left|\mathscr{H}\left(\mathbf{r}_{o}-h \mathbf{q}\right)\right| n\right\rangle}{2 h} .
$$

We thus construct a matrix $W$ for each of the 156 vibrations included in the model and apply our previously described clustering algorithm on them. We find that in order to obtain converged results, a minimum of six clusters is required, schematically depicted in Fig. 3.

The above concludes the necessary steps for parametrizing the linear vibronic Hamiltonian of Eq. (7) for DT-Mes. We now proceed to use this Hamiltonian for a time evolution of the system.

\section{RESULTS}

\section{A. Band-edge excitation}

We start by discussing the population dynamics upon bandedge excitation of the two excitons, i.e., with the system excited and the vibrational modes in their ground state. Figure 6 visualizes these population dynamics. For excitation of the LE state, we barely see any CT formation. On the other hand, exciting the higherenergy CT state leads to relaxation toward LE, within a time scale of approximately 1 ps.

In molecular systems such as the one studied here, the CT state is typically dark, ${ }^{60}$ so exciting it directly is not possible. Instead, one has to excite a "hot" LE configuration, i.e., excite LE beyond its bandedge, giving excess energy to molecular vibrations. This can open a channel to CT formation. To identify specific vibrations which couple the LE and CT excitons, we plot in Fig. 7 the amplitude of modes displaced by $Q=0.2$ or more over the course of the relaxation from CT to LE. We find that a low-frequency mode, with a frequency of

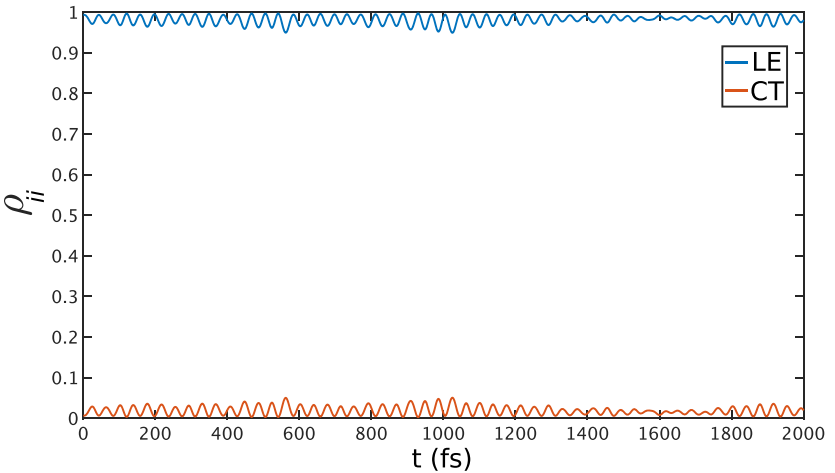

(a)

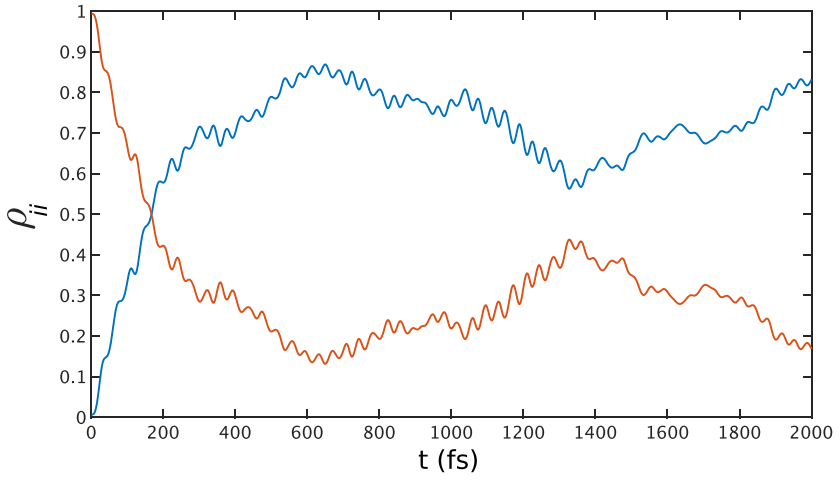

(b)

FIG. 6. Population of the electronic states in real-time, in the cases of initially excited LE and CT states.

$\omega=276 \mathrm{~cm}^{-1}$, is the most active motion. Regarding the real-space motion of this mode, it involves a strong oscillation of the central bond connecting the two tetracene monomers. The strong displacement of this mode upon the CT to LE conversion makes it a good candidate to excite, in order to study the effects of excess energy on $\mathrm{CT}$ formation. From now on, we refer to this vibration simply as "the low-frequency mode." Its coupling constant appearing in the linear vibronic Hamiltonian of Eq. (7) is equal to $\lambda=-289 \mathrm{~cm}^{-1}$.

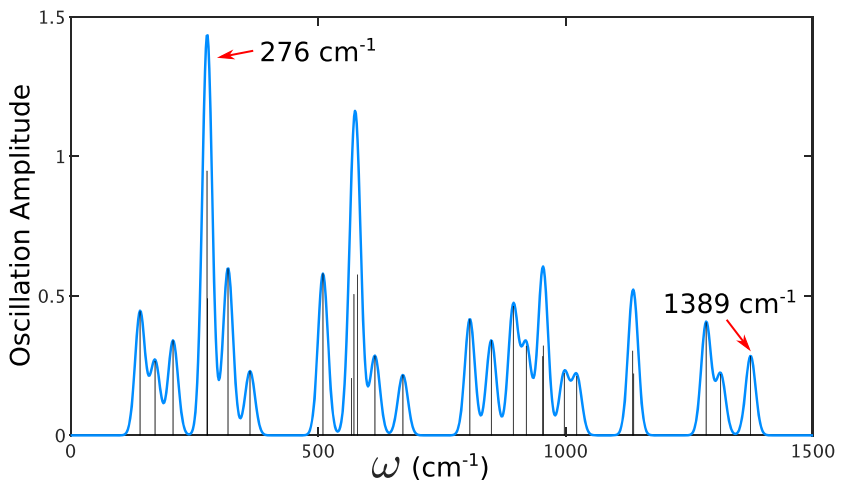

FIG. 7. Amplitude of the most displaced molecular vibrations, during 2 ps of dynamics following CT excitation. 
Furthermore, in order to compare the effects of adding excess energy to the system through a high- and a low-frequency mode, we choose the vibration at $\omega=1389 \mathrm{~cm}^{-1}$ as another candidate for excess energy excitation. We refer to this mode as "the highfrequency mode." This is a breathing motion of the tetracene carbon rings. Its coupling constant is $\lambda=256 \mathrm{~cm}^{-1}$, a value not very far from the one of the low-frequency modes introduced above. We can hence be confident that any differences between the cases of exciting the low- and high-frequency modes are not due to differences in the magnitude of the exciton-phonon coupling constants. The two modes also belong to the same group of modes from the clustering algorithm, so the pattern of their coupling expressed through the matrix $W$ of Eq. (7) is identical.

\section{B. Incoherent excitation}

For the case of adding a set number of quanta to a Fock state of the original vibrations of the molecular system, we only find very small effects on the CT population within 2 ps of dynamics. This is shown for the case of adding quanta to the low- and high-frequency modes in Fig. 8. However, as we show in Sec. III C, this is not the case for coherent excitation of a normal mode, underlining the fact that adding excess energy to a system is not enough by itself, but it needs to be done in the right way in order to facilitate processes of interest.

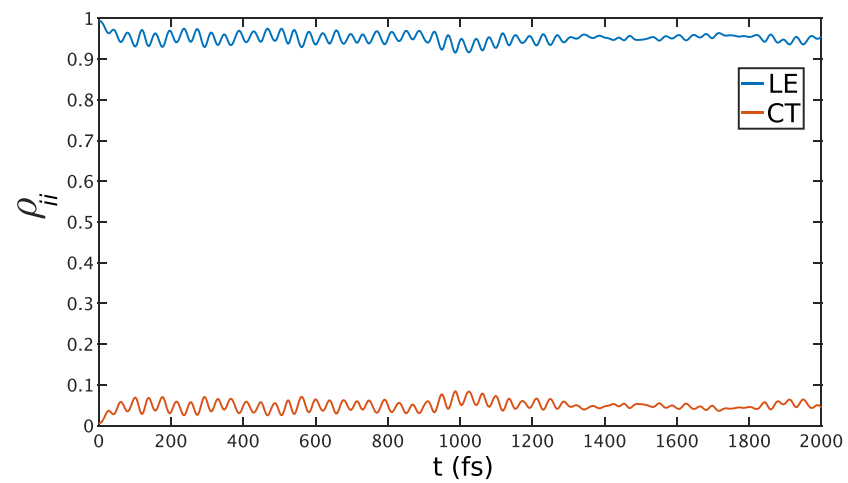

(a)

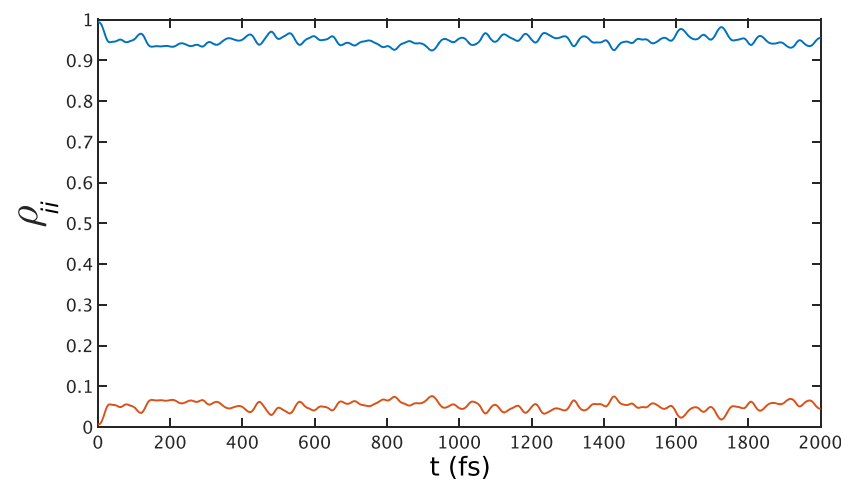

(b)

FIG. 8. Population dynamics upon LE excitation with excess energy added in the system incoherently, through (a) three quanta of a high-frequency mode $(\approx 0.51$ $\mathrm{eV}$ ) and (b) seven quanta of a low-frequency mode $(\approx 0.24 \mathrm{eV})$.

\section{Coherent excitation}

For the case of exciting the two modes coherently as presented in Sec. II, we start by visualizing the population of the two exciton states. Figure 9 shows the resulting dynamics, once the highand low-frequency modes are displaced by $Q=4$. From the linear vibronic Hamiltonian of Eq. (7), it becomes apparent that for the same displacement, the high-frequency mode carries significantly more energy than the low-frequency vibration. For $Q=4$, the two modes carry $0.27 \mathrm{eV}$ and $1.36 \mathrm{eV}$ of energy, respectively.

Comparing the two graphs of Fig. 9, we see that in both cases excess energy opens a channel to CT formation, unlike the case of incoherent excitation summarized in Fig. 8. It therefore becomes clear that in order for excess energy excitation to have an effect on the population dynamics of the two-level system, one needs to induce a coherent displacement of vibrational modes, i.e., excite a wavepacket with finite displacement $Q$. The dynamics toward this CT formation can take longer than the 2 ps window that we have examined thus far, and we therefore simulated the system for a total of $4.5 \mathrm{ps}$ in the cases of coherent excitation.

Furthermore, the two cases of coherent excitation in Fig. 9 exhibit some striking differences, both at early and at later times. Let us start by discussing the early-time $(<100 \mathrm{fs})$ differences between

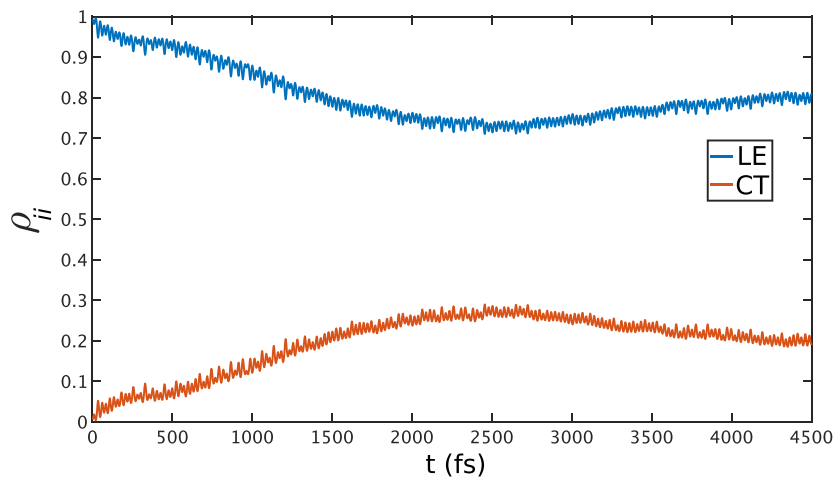

(a)

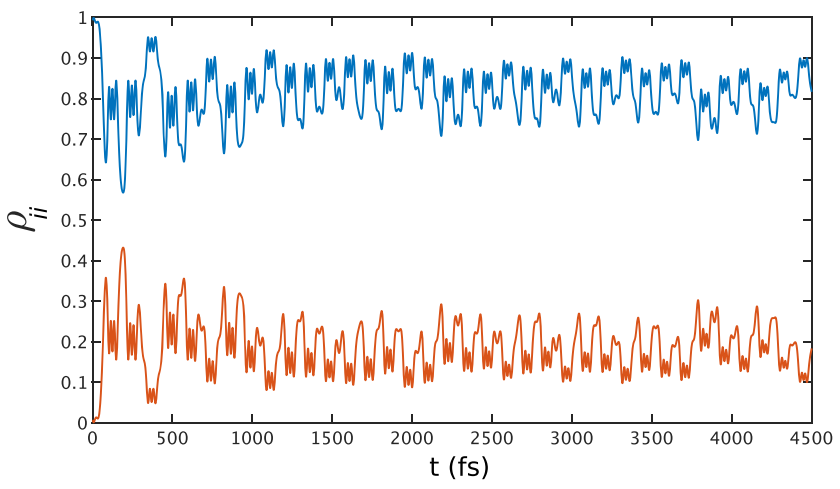

(b)

FIG. 9. Population dynamics upon LE excitation with excess energy added in the system coherently, through (a) a high- and (b) a low-frequency mode vibration. A dimensionless displacement $Q=4$ was chosen for both vibrations, corresponding to excess energy of $1.36 \mathrm{eV}$ and $0.27 \mathrm{eV}$, respectively. 
the two cases. Figure 10 provides a close view of the CT population during these first $100 \mathrm{fs}$ of dynamics. We observe that exciting the low-frequency mode leads to a stronger increase in the CT population (approximately 35\% of CT at $90 \mathrm{fs}$ ), what seems to be an almost constant population growth. Instead, in the high-frequency case, the CT population is oscillatory and does not go beyond $5 \%$. We believe this difference to be due to the time which is required for the crossing event from the LE to the CT surface to occur. The rate of the LE to CT population transfer is dictated by the off-diagonal entries of the linear vibronic Hamiltonian of Eq. (7). All vibrational modes contribute to this value through the linear term; however, it is the electronic coupling of $14 \mathrm{meV}$ which appears in the electronic part of the Hamiltonian [see Eq. (24)] that dominates. This electronic coupling dictates a time scale of approximately $300 \mathrm{fs}$ for the crossing event from the LE to the CT surface, a time scale which is likely to be slightly faster when accounting for the effect of all modes. The period of the high-frequency mode is equal to $24 \mathrm{fs}$; therefore, the wavefunction is only in the vicinity of the crossing for a short time, compared to the low-frequency mode which has a period of $120 \mathrm{fs}$. As a result, only minor population transfer can occur during one period of the high-frequency mode, unlike the low-frequency case where the wavefunction develops significant CT characteristics at these early time scales. This time scale-based argument is further supported by the fact that the energetics of the two excited states are almost identical along the high- and low-frequency

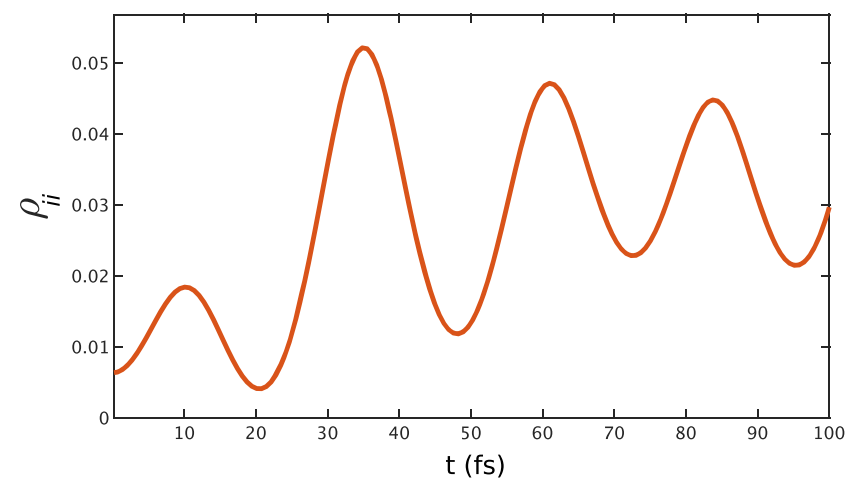

(a)

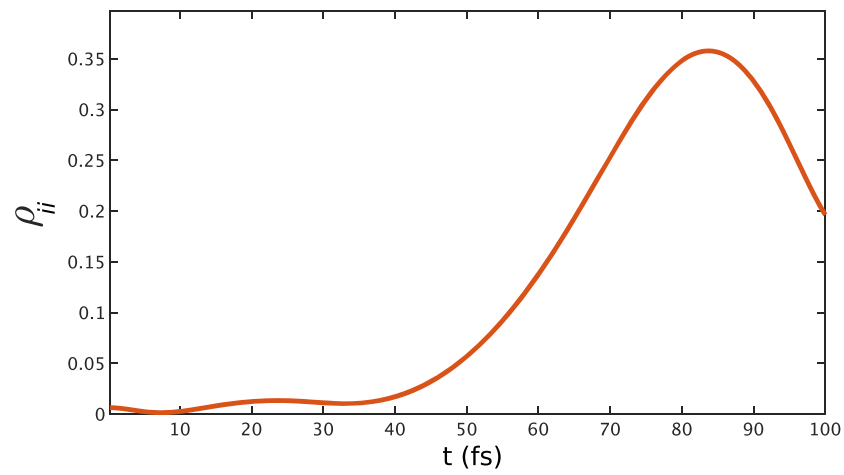

(b)

FIG. 10. Population of the CT state at early times, once excess energy is added in (a) a high-frequency and (b) a low-frequency mode. modes: indeed, from the linear vibronic Hamiltonian of Eq. (7), it becomes obvious that along modes that share the same coupling matrix $W_{i}$ and have similar $\lambda$ values, the energy gap between the excited states is always similar and cannot explain the observed differences.

Moving our focus to the long-time dynamics and back to Fig. 9, for the excitation of the high-frequency mode, the dynamics are dissipative, with a gradual CT formation over approximately $2.5 \mathrm{ps}$. In the low-frequency case, the rapid CT formation at early times is followed by coherent oscillations, with a long damping time scale. Even at $4.5 \mathrm{ps}$, coherent oscillations are still present. Despite the fact that the excess energy used to excite the high-frequency mode surpasses the one used to excite the low-frequency mode by more than $1 \mathrm{eV}$, we see that the final yields of CT population are very close.

We would now like to further understand these long-time results. For both high- and low-frequency mode excitation, we plot in Fig. 11 the charge transfer yield at 2 ps of dynamics, for a range of values of the excess energy. The range of energies is limited by the maximum mode displacement $Q$ we can simulate, related to the maximum bond dimension of the local Hilbert space of the MPS tensors (see Sec. II). This is why for the case of low-frequency modes, the maximum excess energy we can simulate is smaller than for high-frequency modes. However, we clearly see from Fig. 11 that exciting the low-frequency mode leads to significantly larger $\mathrm{CT}$ yields compared to the high-frequency case, at the same excess energy.

The qualitative differences between the long-time population dynamics of Fig. 9 (dissipative vs coherent oscillations) motivate us to plot the quantum coherence between the two exciton states in real-time. The coherences in Figs. 12(b) and 12(c) are clearly different, and we also plot the coherence for band-edge excitation for comparison in Fig. 12(a). For the excitation of a low-frequency mode, the coherence oscillations have an amplitude close to 0.4 (a maximally entangled state has a value of 0.5 ) and are only damped very slowly. When exciting a high-frequency mode, the coherence between the exciton states is more strongly damped and never goes

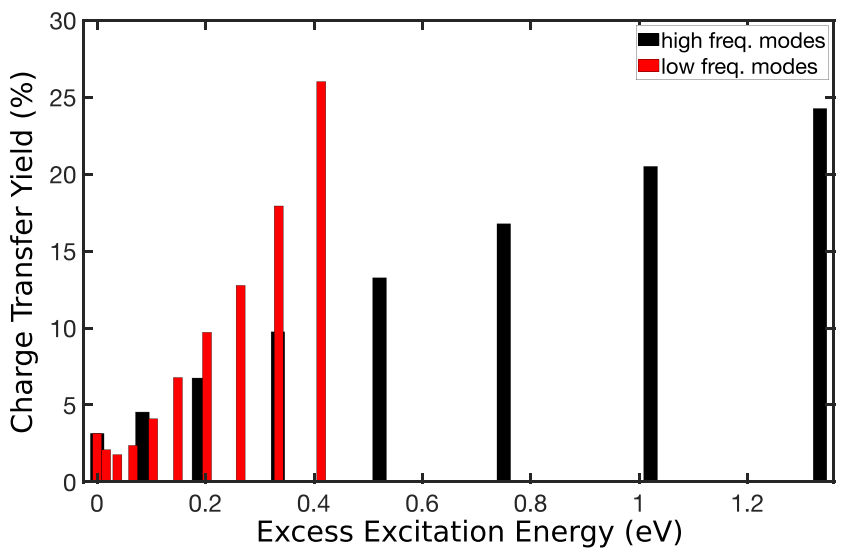

FIG. 11. Charge transfer yield at 2 ps following photoexcitation, for various values of the excitation energy, which is pumped into the system through low- or high-frequency modes. All the presented data refer to coherent excitation. For incoherent excitation, yields never surpass $5 \%-6 \%$. 


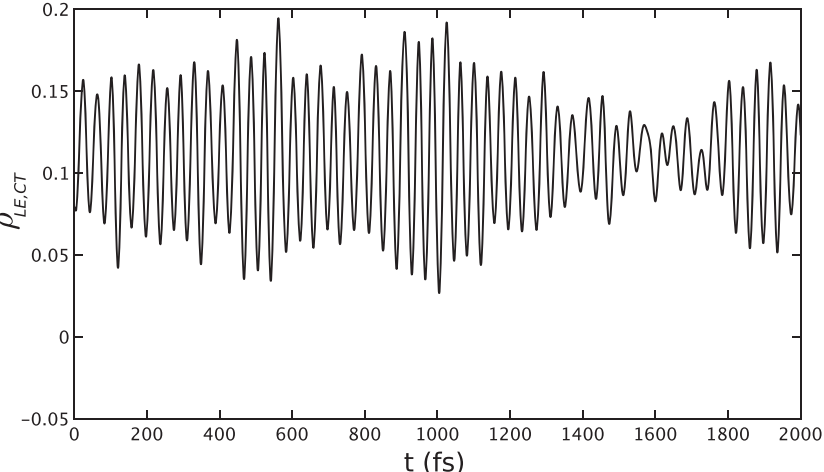

(a)

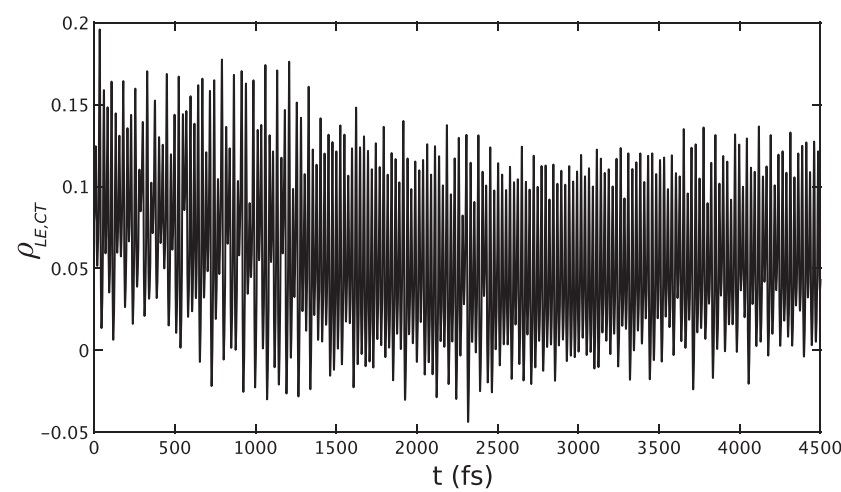

(b)

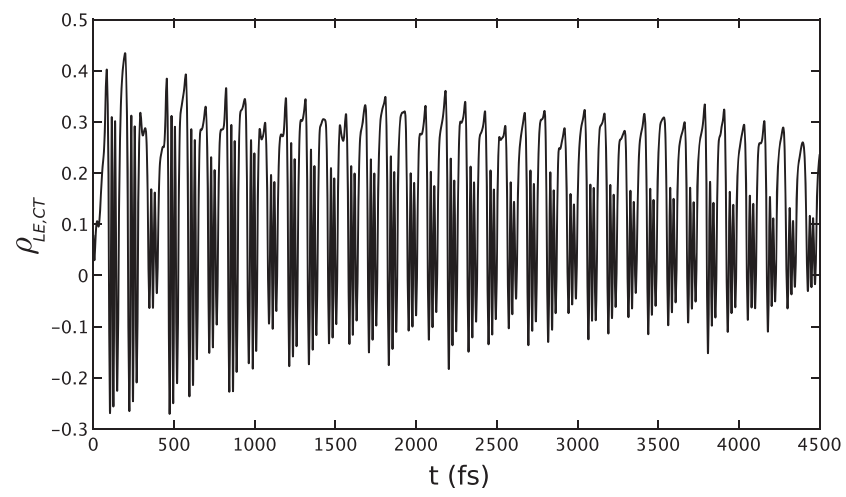

(c)

FIG. 12. Quantum coherence between the LE and CT states for (a) band-edge excitation and excess energy excitation through (b) a high- and (c) a low-frequency mode.

beyond a value of 0.2 , even at early times. The dissipation of coherence ceases at approximately $2.5 \mathrm{ps}$, when the charge transfer process of Fig. 9(a) is mostly complete. No such coherence dissipation is observed in Fig. 12(a), where no excess energy is present. This leads us to the conclusion that the dissipation of coherence at long times is related to the vibrational relaxation which follows excess energy excitation. However, the differences between Figs. 12(b) and 12 (c) point to the fact that this relaxation process can be significantly different, depending on whether a low- or a high-frequency vibration was initially excited, as no significant coherence dissipation is present for the low-frequency case. The relaxation pathway of the system seems to have a direct impact on its ability to maintain a superposition state at long times, with the excitation of a low-frequency mode "protecting" the quantum coherence between the two excitons and allowing for a more efficient energy transfer. However, the microscopic difference for this different coherence behavior is still not clear at this stage.

A microscopic understanding of the different coherencerelated properties may be obtained by considering the details of the vibrational relaxation process. Once we excite a high-frequency (i.e., high-energy) mode, the system will start to relax toward a Boltzmann distribution, where low-energy vibrations are predominantly displaced. Within our model picture, we expect the amplitude of low-frequency vibrations to increase with time. Indeed, as we find in Fig. 13(a), the most significant increase in vibrational amplitude $\Delta \mathrm{Q}_{\max }$ over the course of 4 ps occurs for low-frequency modes. As discussed, when considering the early-time differences between exciting low-frequency and high-frequency modes, low-frequency vibrations facilitate more efficient crossings from the LE to the CT surface. Therefore, the gradual vibrational relaxation toward lowfrequency modes coincides with the increase in the CT population observed in Fig. 9(a). There is little change in the amplitudes of

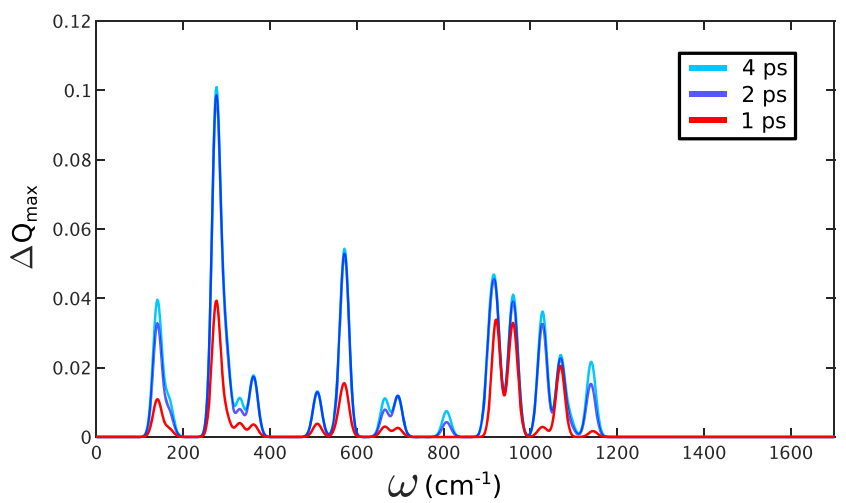

(a)

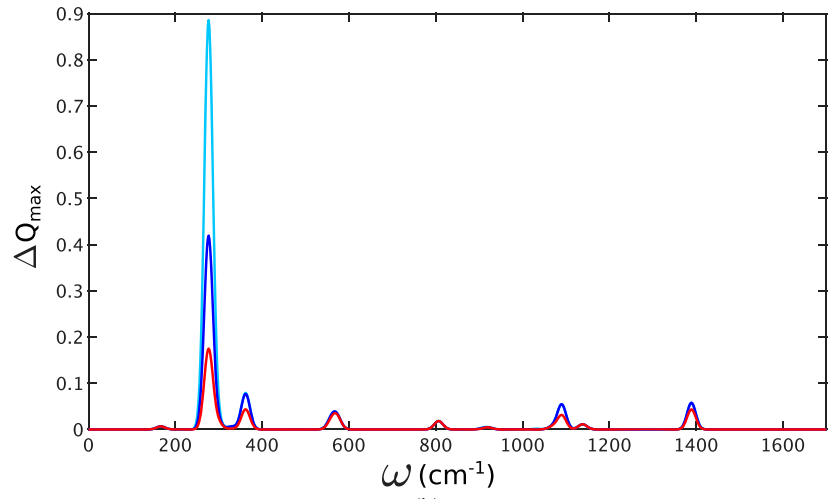

(b)

FIG. 13. Vibrational relaxation upon excitation of (a) the high-frequency and (b) the low-frequency mode. $\Delta Q_{\max }$ denotes the change in mode amplitudes over the studied time scales. 
vibrations between 2 ps and 4 ps, suggesting that vibrational relaxation is mostly complete. Indeed, the population of the CT state also reaches its maximum in these time scales.

Overall, there is a large number of vibrations toward which the excess energy relaxes once we excite a high-frequency mode. However, we note that our model only includes an implicit anharmonicity; the modes are only coupled to each other through the electronic system and not explicitly, making the transfer of the vibrational energy slower than if mode-mode energy transfer was included in our description. It is crucial in order to obtain realistic time scales for vibrational relaxation to include explicit anharmonicity in a model description. With the present approach, this would pose a significant challenge, as the coupling between vibrational modes would give rise to highly nonlocal interactions between MPS tensors. We therefore comment on the qualitative characteristics of the vibrational relaxation process, rather than the quantitative time scales.

The change in mode amplitudes looks very different, once excess energy excites a low-frequency vibration in Fig. 13(b). The distribution of excited modes is now much more localized around low frequencies. The mode which gets strongly excited is degenerate with our low-frequency vibration of choice within $1 \mathrm{~cm}^{-1}$, so excess energy remains essentially "trapped" (the modes of molecular dimers such as the studied one always go in pairs). Overall, only a small number of other oscillators are activated within $4 \mathrm{ps.}$

The above observations allow us to build a mechanical analog, which rationalizes the process of vibrational relaxation and helps understand the differences between low- and high-frequency mode excitation at long times. This is visualized in Fig. 14. Jars of various sizes are placed in a gravitational field, connected to each other through a system of pipes. Larger jars are placed higher up in the field, causing an efficient water flow toward lower-lying jars. By initially placing all of the available water in one of the high-lying jars, we end up at intermediate times (i.e., before time scales relevant at the Boltzmann equilibrium), with a wide distribution of water over the low-lying jars.

On the other hand, if we place all of the available water in a low-lying jar, it can to some extent flow uphill through the pipe system; however, this is rather inefficient because of gravity. Also, the

\section{High-frequency mode excitation}
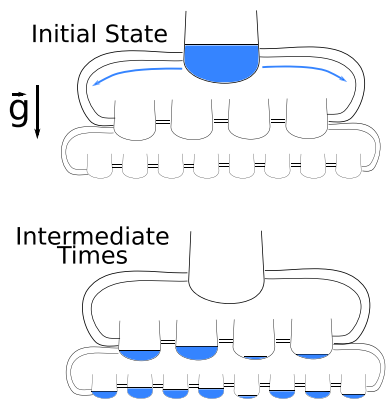

FIG. 14. A mechanical analog to our model for vibrational relaxation. A system of jars of different sizes, filled with water in a gravitational field, and communicating through a system of pipes. flow to other low-lying jars is not at all affected by the presence of the gravitational field. Hence, for intermediate time scales, we have a distribution of water which is much more localized compared to the case where we put all of the water in a high-lying jar. We emphasize here that both initial conditions will eventually lead to a Boltzmann equilibrium.

If we now substitute "water" with energy, "jars" of various sizes with modes of different frequencies, "pipes" with mode-mode coupling (anharmonicity), and "gravity" with energy gradient, we get a model description of vibrational relaxation. Exciting a highfrequency mode results in relaxation toward lower energy vibrations, a process which is assisted by the existence of an energy gradient. Therefore, for intermediate time scales from adding excess energy to a high-frequency mode, we end up with a wide distribution of the energy over vibrational modes, predominantly of lowfrequencies, as seen in Fig. 13(a). Once any of these oscillators is excited, it has a random phase, making it more and more difficult for the total wavefunction to maintain any well-defined phase initially present. Therefore, the quantum coherence between the involved exciton states dissipates away. This becomes clear by comparing the slowly vanishing coherence of Fig. 12(b) to that of the band-edge excitation dynamics in Fig. 12(a). These intermediate configurations resulting from high-frequency mode excitation are more incoherent and approach the limit of incoherent excitation studied in Sec. III B.

For excitation of a low-frequency vibration, the limited pathways toward other modes lead to a more localized distribution of the excess energy at intermediate time scales, as visualized in Fig. 13(b). It is therefore possible at these intermediate time scales to maintain the phase initially present in the system. Hence, the quantum coherence between the exciton states is "protected" for these intermediate time scales relevant to ultrafast processes, as seen in Fig. 12(c).

\section{CONCLUSIONS}

In this work, we have exhibited the implementation of a theoretical framework, which allows us to simulate the ultrafast dynamics of organic structures upon photoexcitation. Our tensor network approach allows us to perform a quantum mechanical time evolution of the vibrational wavefunction, which in turn makes it possible to visualize the real-time motion with atomistic level of detail. We have also developed methods to simulate the effects of excess energy excitation within the context of matrix product states.

We applied our methods to a covalently linked tetracene dimer, studying intramolecular charge transfer, a process which is endothermic in this system. Not only have we found that the final yield of charge transfer states strongly depends on the excitation energy of the light pulse, but also on the way this excess energy is introduced into the system. In particular, coherent excitation of a vibrational wavepacket opens a channel to charge transfer, whereas incoherent excitation only has a minor effect. We found the coherent excitation of low-frequency modes to be a much more efficient way of enhancing charge transfer compared to the excitation of highfrequency modes. This is due to the different pathways of vibrational relaxation in the two cases; low-frequency vibrations have a more limited number of pathways toward a Boltzmann equilibrium, leading to a more localized distribution of excited oscillators in the 
intermediate time scales relevant for charge transfer. Thus, the quantum coherence between the bright and charge transfer states is better preserved compared to the case of exciting high-frequency modes, where we found the dynamics to be more dissipative, approaching the limit of incoherent excitation.

Experimentally, the effect of mode-selective excitation on ultrafast processes has been demonstrated in the past. ${ }^{44,45}$ We believe that our study of the underlying mechanisms of vibrational relaxation following excess energy excitation could provide a guideline for future experimental work in this field, potentially focusing on exploiting specific vibrations for enhancing the efficiency of processes utilized in devices.

Our results for the studied two-level system are general and will hold for any two-level system coupled with a bath of oscillators. As the system relaxes toward its Boltzmann equilibrium, the initial configuration, and in particular the part of the system where excess energy may initially be localized, may have a strong effect on the properties at intermediate time scales. In particular, initial configurations with excess energy in low-frequency vibrations lie closer to the final Boltzmann distribution, reducing the number of pathways the system may follow toward equilibrium.
Hence, low-frequency modes preserve quantum coherence more efficiently, making them better candidates for the transfer of information.

\section{ACKNOWLEDGMENTS}

The authors would like to acknowledge the support of the Winton Programme for the Physics of Sustainability and to thank Akshay Rao, Timothy J. H. Hele, Andrew J. Musser, and Richard H. Friend for useful discussions. A.M.A. acknowledges the support of the Engineering and Physical Sciences Research Council (EPSRC) for funding under Grant No. EP/L015552/1.

\section{APPENDIX: CONVERGENCE TESTS}

We study the convergence of the dynamics simulations in respect of the bond dimensions of the MPS. There are two separate bond dimensions to consider: The first is the intrachain bond dimension (D), which encodes the entanglement between the environment tensors, while the second $\left(D_{h}\right)$ refers to the system-environment coupling (see Fig. 2). We use the yield of the

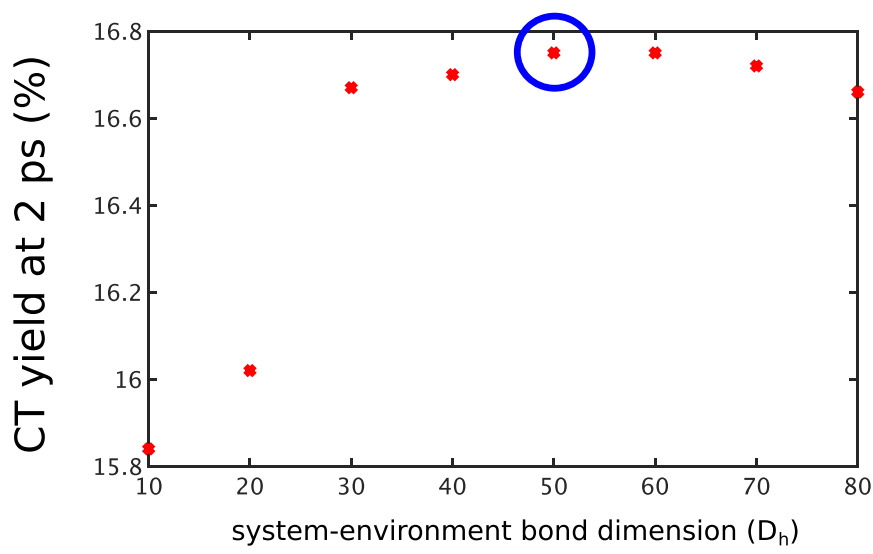

(a)

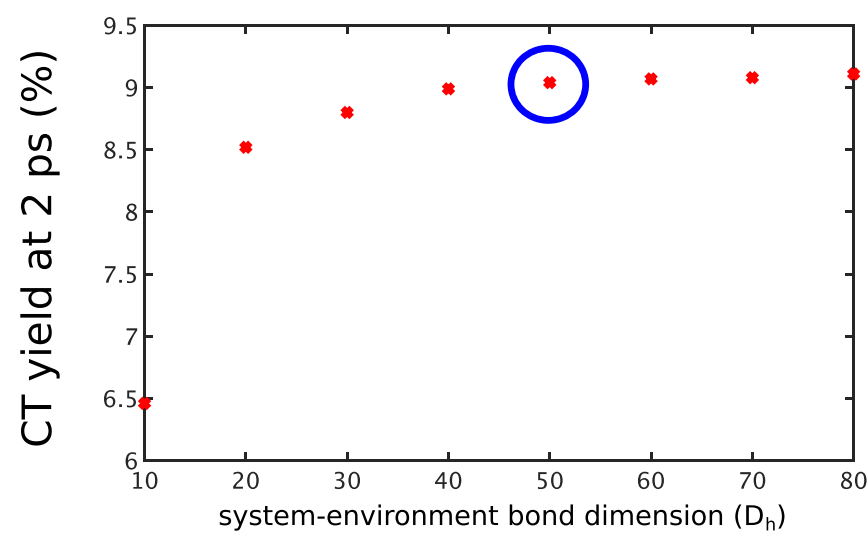

(c)

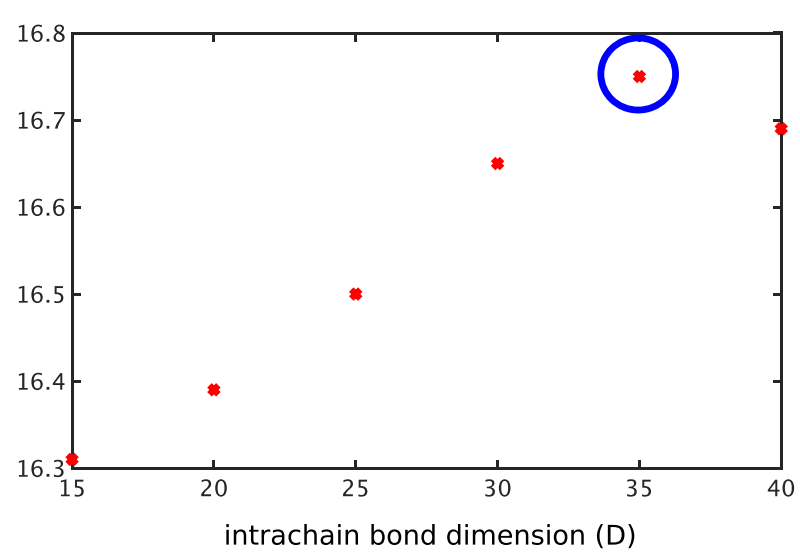

(b)

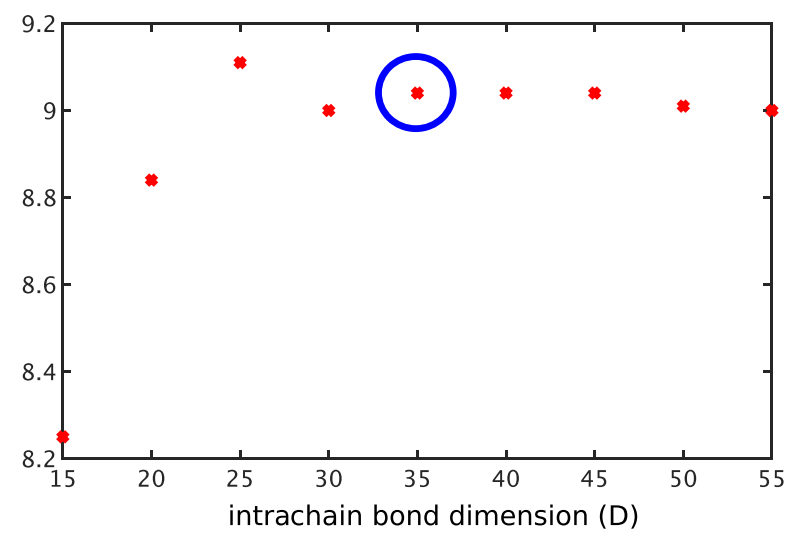

(d)

FIG. 15. Convergence of CT populations at $2 \mathrm{ps}$ with respect to bond dimensions for band-edge and excess-energy excitation. 


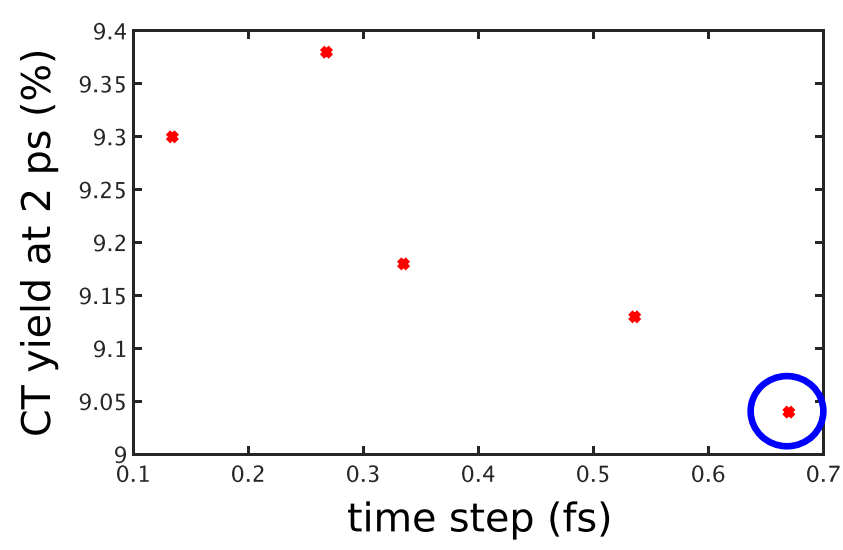

FIG. 16. Convergence of CT populations at $2 \mathrm{ps}$ with respect to the simulation time step for the case of excess energy excitation of the high-frequency mode.

CT state at 2 ps as an indicator for convergence. We study the convergence of dynamics following band-edge excitation of the CT state [akin to Fig. 6(b)], in Fig. 15(a). The values which were used in the main part of the paper are highlighted. We have also examined the convergence in the case of adding excess energy to the high-frequency vibrations, displacing it by $Q=2$, and the results are shown in Fig. 15(b).

We have also tested the convergence of the dynamics following excess energy excitation of the high-frequency mode, in respect of the time step of the simulation. The results are shown in Fig. 16. We use a time step of $0.67 \mathrm{fs}$, despite the fact that this value is not perfectly converged. This allows us to significantly speed up our calculations and investigate the results at longer time scales. Compared to the smallest value for the time step of the simulations, the error in the CT yield is smaller than $0.3 \%$.

\section{REFERENCES}

${ }^{1}$ R. Blankenship, Molecular Mechanisms of Photosynthesis (Wiley-Blackwell, 2014).

${ }^{2}$ H. v. Amerongen, L. Valkunas, and R. v. Grondelle, Photosynthetic Excitons (World Scientific, 2000).

${ }^{3}$ B. Kippelen and J.-L. Bredas, "Organic photovoltaics," Energy Environ. Sci. 2, 251-261 (2008).

${ }^{4}$ E. J. O'Reilly and A. Olaya-Castro, "Non-classicality of the molecular vibrations assisting exciton energy transfer at room temperature," Nat. Commun. 5, 3012 (2014).

${ }^{5}$ J. Cabanillas-Gonzalez, G. Grancini, and G. Lanzani, "Pump-probe spectroscopy in organic semiconductors: Monitoring fundamental processes of relevance in optoelectronics," Adv. Mater. 23, 5468-5485 (2011).

${ }^{6}$ S. Gélinas, A. Rao, A. Kumar, S. L. Smith, A. W. Chin, J. Clark, T. S. van der Poll, G. C. Bazan, and R. H. Friend, "Ultrafast long-range charge separation in organic semiconductor photovoltaic diodes," Science 343, 512-516 (2014).

${ }^{7}$ S. M. Falke, C. A. Rozzi, D. Brida, M. Maluri, M. Amato, E. Sommer, A. De Sio, A. Rubio, G. Cerullo, E. Molinari, and C. Lienau, "Coherent ultrafast charge transfer in an organic photovoltaic blend," Science 344, 1001-1005 (2014). ${ }^{8}$ C. Y. Wong, S. B. Penwell, B. L. Cotts, R. Noriega, H. Wu, and N. S. Ginsberg, "Revealing exciton dynamics in a small-molecule organic semiconducting film with subdomain transient absorption microscopy," J. Phys. Chem. C 117, 22111-22122 (2013).
${ }^{9}$ Y. Wan, Z. Guo, T. Zhu, S. Yan, J. Johnson, and L. Huang, "Cooperative singlet and triplet exciton transport in tetracene crystals visualized by ultrafast microscopy," Nat. Chem. 7, 785-792 (2015).

${ }^{10}$ S. L. Smith and A. W. Chin, "Phonon-assisted ultrafast charge separation in the PCBM band structure,” Phys. Rev. B 91, 201302 (2015).

${ }^{11}$ A. A. Bakulin, S. E. Morgan, T. B. Kehoe, M. W. B. Wilson, A. W. Chin, D. Zigmantas, D. Egorova, and A. Rao, "Real-time observation of multiexcitonic states in ultrafast singlet fission using coherent $2 \mathrm{D}$ electronic spectroscopy," Nat. Chem. 8, 16-23 (2016).

${ }^{12}$ A. J. Musser, M. Liebel, C. Schnedermann, T. Wende, T. B. Kehoe, A. Rao, and P. Kukura, "Evidence for conical intersection dynamics mediating ultrafast singlet exciton fission,” Nat. Phys. 11, 352-357 (2015).

${ }^{13}$ R. Tempelaar and D. R. Reichman, "Vibronic exciton theory of singlet fission. I. Linear absorption and the anatomy of the correlated triplet pair state," J. Chem. Phys. 146, 174703 (2017); e-print arXiv:1703.01173.

${ }^{14}$ A. A. Bakulin, A. Rao, V. G. Pavelyev, P. H. M. van Loosdrecht, M. S. Pshenichnikov, D. Niedzialek, J. Cornil, D. Beljonne, and R. H. Friend, "The role of driving energy and delocalized states for charge separation in organic semiconductors," Science 335, 1340-1344 (2012).

${ }^{15}$ G. Grancini, M. Maiuri, D. Fazzi, A. Petrozza, H. J. Egelhaaf, D. Brida, G. Cerullo, and G. Lanzani, "Hot exciton dissociation in polymer solar cells," Nat. Mater. 12, 29-33 (2013).

${ }^{16}$ J.-L. Brédas, E. H. Sargent, and G. D. Scholes, "Photovoltaic concepts inspired by coherence effects in photosynthetic systems," Nat. Mater. 16, 35 (2017).

${ }^{17}$ G. D. Scholes, G. R. Fleming, L. X. Chen, A. Aspuru-Guzik, A. Buchleitner, D. F. Coker, G. S. Engel, R. Van Grondelle, A. Ishizaki, D. M. Jonas et al., "Using coherence to enhance function in chemical and biophysical systems," Nature 543, 647 (2017).

${ }^{18}$ C. Creatore, M. A. Parker, S. Emmott, and A. W. Chin, "Efficient biologically inspired photocell enhanced by delocalized quantum states," Phys. Rev. Lett. 111, 253601 (2013).

${ }^{19}$ M. Sarovar, A. Ishizaki, G. R. Fleming, and K. B. Whaley, "Quantum entanglement in photosynthetic light-harvesting complexes," Nat. Phys. 6, 462-467 (2010).

${ }^{20}$ D. Bacon, K. R. Brown, and K. B. Whaley, "Coherence-preserving quantum bits,” Phys. Rev. Lett. 87, 247902-247902-4 (2001).

${ }^{21}$ A. W. Chin, J. Prior, R. Rosenbach, F. Caycedo-Soler, S. F. Huelga, and M. B. Plenio, "The role of non-equilibrium vibrational structures in electronic coherence and recoherence in pigment-protein complexes," Nat. Phys. 9, 113-118 (2013); e-print arXiv:1203.0776.

${ }^{22}$ J. Roden, W. T. Strunz, K. B. Whaley, and A. Eisfeld, "Accounting for intramolecular vibrational modes in open quantum system description of molecular systems,"J. Chem. Phys. 137, 204110 (2012).

${ }^{23}$ E. R. Bittner and C. Silva, "Noise-induced quantum coherence drives photocarrier generation dynamics at polymeric semiconductor heterojunctions," Nat. Commun. 5, 3119 (2014); e-print arXiv:1309.6975.

${ }^{24}$ T. C. Berkelbach, M. S. Hybertsen, and D. R. Reichman, "Microscopic theory of singlet exciton fission. I. General formulation,” J. Chem. Phys. 141, 074705 (2014); e-print arXiv: $1211.6458 \mathrm{v} 1$.

${ }^{25}$ F. Mirjani, N. Renaud, N. Gorczak, and F. C. Grozema, "Theoretical investigation of singlet fission in molecular dimers: The role of charge transfer states and quantum interference," J. Phys. Chem. C 118, 14192-14199 (2014).

${ }^{26}$ M. Zarea, D. Powell, N. Renaud, M. R. Wasielewski, and M. A. Ratner, "Decoherence and quantum interference in a four-site model system: Mechanisms and turnovers," J. Phys. Chem. B 117, 1010-1020 (2013).

${ }^{27}$ J. Zheng, Y. Xie, S. Jiang, and Z. Lan, "Ultrafast nonadiabatic dynamics of singlet fission: Quantum dynamics with the multilayer multiconfigurational timedependent hartree (ML-MCTDH) method," J. Phys. Chem. C 120, 1375-1389 (2016).

${ }^{28}$ R. Binder, D. Lauvergnat, and I. Burghardt, "Conformational dynamics guides coherent exciton migration in conjugated polymer materials: First-principles quantum dynamical study," Phys. Rev. Lett. 120, 227401 (2018).

${ }^{29}$ N. R. Monahan, D. Sun, H. Tamura, K. W. Williams, B. Xu, Y. Zhong, B. Kumar, C. Nuckolls, A. R. Harutyunyan, G. Chen, H. L. Dai, D. Beljonne, Y. Rao, and X. Y. Zhu, "Dynamics of the triplet-pair state reveals the likely coexistence of 
coherent and incoherent singlet fission in crystalline hexacene," Nat. Chem. 9, 341-346 (2017).

${ }^{30}$ B. Monserrat, E. A. Engel, and R. J. Needs, "Giant electron-phonon interactions in molecular crystals and the importance of nonquadratic coupling," Phys. Rev. B 92, 140302 (2015).

${ }^{31}$ Y. Y. Shi, L. M. Duan, and G. Vidal, "Classical simulation of quantum manybody systems with a tree tensor network," Phys. Rev. A 74, 022320 (2006).

${ }^{32} \mathrm{U}$. Schollwöck, "The density-matrix renormalization group in the age of matrix product states," Ann. Phys. 326, 96-192 (2011).

${ }^{33}$ F. A. Y. N. Schröder, D. H. P. Turban, A. J. Musser, N. D. M. Hine, and A. W. Chin, "Tensor network simulation of multi-environmental open quantum dynamics via machine learning and entanglement renormalisation," Nat. Commun. 10, 1062 (2019).

${ }^{34}$ J. Del Pino, F. A. Schröder, A. W. Chin, J. Feist, and F. J. Garcia-Vidal, "Tensor network simulation of polaron-polaritons in organic microcavities," Phys. Rev. B 98, 165416 (2018); e-print arXiv: 1807.00586.

${ }^{35}$ J. del Pino, F. A. Y. N. Schröder, A. W. Chin, J. Feist, and F. J. Garcia-Vidal, "Tensor network simulation of non-Markovian dynamics in organic polaritons," Phys. Rev. Lett. 121, 227401 (2018); e-print arXiv:1804.04511.

${ }^{36} \mathrm{H}$. Wang and $\mathrm{M}$. Thoss, "Multilayer formulation of the multiconfiguration time-dependent Hartree theory," J. Chem. Phys. 119, 1289-1299 (2003).

${ }^{37}$ K. Vandewal, S. Albrecht, E. T. Hoke, K. R. Graham, J. Widmer, J. D. Douglas, M. Schubert, W. R. Mateker, J. T. Bloking, G. F. Burkhard, A. Sellinger, J. M. J. Fréchet, A. Amassian, M. K. Riede, M. D. McGehee, D. Neher, and A. Salleo, "Efficient charge generation by relaxed charge-transfer states at organic interfaces," Nat. Mater. 13, 63-68 (2014).

${ }^{38}$ J. Kurpiers, T. Ferron, S. Roland, M. Jakoby, T. Thiede, F. Jaiser, S. Albrecht, S. Janietz, B. A. Collins, I. A. Howard, and D. Neher, "Probing the pathways of free charge generation in organic bulk heterojunction solar cells," Nat. Commun. 9, 2038 (2018)

${ }^{39}$ A. J. Musser, M. Al-Hashimi, M. Maiuri, D. Brida, M. Heeney, G. Cerullo, R. H. Friend, and J. Clark, "Activated singlet exciton fission in a semiconducting polymer," J. Am. Chem. Soc. 135, 12747-12754 (2013).

${ }^{40}$ D. Paleček, P. Edlund, S. Westenhoff, and D. Zigmantas, "Quantum coherence as a witness of vibronically hot energy transfer in bacterial reaction center," Sci. Adv. 3, el603141 (2017).

${ }^{41}$ D. N. Beratan and D. H. Waldeck, "DNA charge transfer: Hot holes break the speed limit," Nat. Chem. 8, 992-993 (2016).

${ }^{42}$ H. Tamura, R. Martinazzo, M. Ruckenbauer, and I. Burghardt, "Quantum dynamics of ultrafast charge transfer at an oligothiophene-fullerene heterojunction," J. Chem. Phys. 137, 22A540 (2012).

${ }^{43}$ B. Kloss, D. Reichman, and R. Tempelaar, "Multi-set matrix product state calculations reveal mobile Franck-Condon excitations under strong Holstein-type coupling," e-print arXiv:1812.00011 (2018).

${ }^{44}$ A. A. Bakulin, R. Lovrincic, X. Yu, O. Selig, H. J. Bakker, Y. L. A. Rezus, P. K. Nayak, A. Fonari, V. Coropceanu, J. L. Brédas, and D. Cahen, "Mode-selective vibrational modulation of charge transport in organic electronic devices," Nat. Commun. 6, 7880 (2015).

${ }^{45}$ M. Delor, T. Keane, P. A. Scattergood, I. V. Sazanovich, G. M. Greetham, M. Towrie, A. J. Meijer, and J. A. Weinstein, "On the mechanism of vibrational control of light-induced charge transfer in donor-bridge-acceptor assemblies," Nat. Chem. 7, 689-695 (2015).

${ }^{46}$ C. Guo, A. Weichselbaum, J. von Delft, and M. Vojta, "Critical and strongcoupling phases in one- and two-bath spin-boson models," Phys. Rev. Lett. 108, 160401 (2012).
${ }^{47}$ F. A. Y. N. Schröder and A. W. Chin, "Simulating open quantum dynamics with time-dependent variational matrix product states: Towards microscopic correlation of environment dynamics and reduced system evolution," Phys. Rev. B 93, 075105 (2016).

${ }^{48}$ A. W. Chin, Á. Rivas, S. F. Huelga, and M. B. Plenio, "Exact mapping between system-reservoir quantum models and semi-infinite discrete chains using orthogonal polynomials," J. Math. Phys. 51, 092109 (2010).

${ }^{49}$ J. Prior, I. de Vega, A. W. Chin, S. F. Huelga, and M. B. Plenio, "Quantum dynamics in photonic crystals," Phys. Rev. A 87, 013428 (2013).

${ }^{50}$ J. Prior, A. W. Chin, S. F. Huelga, and M. B. Plenio, "Efficient simulation of strong system-environment interactions," Phys. Rev. Lett. 105, 050404 (2010).

${ }^{51}$ D. Arthur and S. Vassilvitskii, "K-means++: The advantages of careful seeding," in Proceedings of the Eighteenth Annual ACM-SIAM Symposium on Discrete Algorithms (Society for Industrial and Applied Mathematics: Philadelphia, PA, 2007), pp. 1027-1035.

${ }^{52}$ L. Van Der Maaten, G. Hinton, and G. H. van der Maaten, "Visualizing Data using t-SNE," J. Mach. Learn. Res. 9, 2579-2605 (2008).

${ }^{53}$ J. Haegeman, C. Lubich, I. Oseledets, B. Vandereycken, and F. Verstraete, "Unifying time evolution and optimization with matrix product states," Phys. Rev. B 94, 165116 (2016).

${ }^{54}$ G. Vidal, "Entanglement renormalization," Phys. Rev. Lett. 99, 220405 (2007).

${ }^{55}$ S. M. Barnett and P. M. Radmore, Methods in Theoretical Quantum Optics, Oxford Series in Optical and Imaging Science Vol. 15 (Clarendon, Oxford, 2005).

${ }^{56}$ M. B. Smith and J. Michl, "Singlet fission," Chem. Rev. 110, 6891-6936 (2010).

${ }^{57}$ A. Rao and R. H. Friend, "Harnessing singlet exciton fission to break the Shockley-Queisser limit,” Nat. Rev. Mater. 2, 17063 (2017).

${ }^{58}$ M. W. Wilson, A. Rao, K. Johnson, S. Gélinas, R. Di Pietro, J. Clark, and R. H. Friend, "Temperature-independent singlet exciton fission in tetracene," J. Am. Chem. Soc. 135, 16680-16688 (2013).

${ }^{59}$ H. L. Stern, A. Cheminal, S. R. Yost, K. Broch, S. L. Bayliss, K. Chen, M. Tabachnyk, K. Thorley, N. Greenham, J. M. Hodgkiss, J. Anthony, M. Head-Gordon, A. J. Musser, A. Rao, and R. H. Friend, "Vibronically coherent ultrafast triplet-pair formation and subsequent thermally activated dissociation control efficient endothermic singlet fission," Nat. Chem. 9, 1205 (2017).

${ }^{60}$ S. Lukman, K. Chen, J. M. Hodgkiss, D. H. P. Turban, N. D. M. Hine, S. Dong, J. Wu, N. C. Greenham, and A. J. Musser, "Tuning the role of charge-transfer states in intramolecular singlet exciton fission through side-group engineering," Nat. Commun. 7, 13622 (2016).

${ }^{61}$ J. A. Pople, "Electron interaction in unsaturated hydrocarbons," Trans. Faraday Soc. 49, 1375 (1953).

${ }^{62}$ R. Pariser and R. G. Parr, "A semi-empirical theory of the electronic spectra and electronic structure of complex unsaturated molecules. I," J. Chem. Phys. 21, 466-471 (1953).

${ }^{63} \mathrm{P}$. Tavan and K. Schulten, "Electronic excitations in finite and infinite polyenes," Phys. Rev. B 36, 4337-4357 (1987).

${ }^{64} \mathrm{~K}$. Aryanpour, A. Shukla, and S. Mazumdar, "Theory of singlet fission in polyenes, acene crystals, and covalently linked acene dimers," J. Phys. Chem. C 119, 6966-6979 (2015).

${ }^{65}$ B. Njegic and M. S. Gordon, "Exploring the effect of anharmonicity of molecular vibrations on thermodynamic properties," J. Chem. Phys. 125, 224102 (2006). 